\title{
Improved observations of turbulence dissipation rates from wind profiling radars
}

\author{
Katherine McCaffrey ${ }^{1,2}$, Laura Bianco ${ }^{1,2}$, and James M. Wilczak ${ }^{2}$ \\ ${ }^{1}$ University of Colorado, Cooperative Institute for Research in Environmental Sciences at the NOAA Earth System Research \\ Laboratory, Physical Sciences Division, 325 Broadway, Boulder, CO 80305-3337, USA \\ ${ }^{2}$ NOAA Earth System Research Laboratory, Physical Sciences Division, 325 Broadway, Boulder, CO 80305-3337, USA \\ Correspondence to: Katherine McCaffrey (katherine.mccaffrey@noaa.gov)
}

Received: 29 September 2016 - Discussion started: 4 October 2016

Revised: 21 June 2017 - Accepted: 22 June 2017 - Published: 20 July 2017

\begin{abstract}
Observations of turbulence dissipation rates in the planetary boundary layer are crucial for validation of parameterizations in numerical weather prediction models. However, because dissipation rates are difficult to obtain, they are infrequently measured through the depth of the boundary layer. For this reason, demonstrating the ability of commonly used wind profiling radars (WPRs) to estimate this quantity would be greatly beneficial. During the XPIA field campaign at the Boulder Atmospheric Observatory, two WPRs operated in an optimized configuration, using high spectral resolution for increased accuracy of Doppler spectral width, specifically chosen to estimate turbulence from a vertically pointing beam. Multiple post-processing techniques, including different numbers of spectral averages and peak processing algorithms for calculating spectral moments, were evaluated to determine the most accurate procedures for estimating turbulence dissipation rates using the information contained in the Doppler spectral width, using sonic anemometers mounted on a $300 \mathrm{~m}$ tower for validation. The optimal settings were determined, producing a low bias, which was later corrected. Resulting estimations of turbulence dissipation rates correlated well $\left(R^{2}=0.54\right.$ and 0.41$)$ with the sonic anemometers, and profiles up to $2 \mathrm{~km}$ from the $449 \mathrm{MHz}$ WPR and $1 \mathrm{~km}$ from the $915 \mathrm{MHz}$ WPR were observed.
\end{abstract}

\section{Introduction}

In the kinetic energy balance of the atmosphere, the components due to turbulent motions can be the most difficult to predict and require parameterizations in numerical models to estimate the contributions at scales smaller than simulations can resolve. Validation of these parameterizations, however, requires observations that are often not available or are limited in time and space. Recent work evaluating the sensitivity of a combined planetary boundary layer (PBL) and surface layer parameterization scheme within the Weather Research and Forecasting (WRF) model has shown that the model forecast skill for boundary layer winds is most sensitive to the parameterization of the turbulent kinetic energy (TKE) dissipation rate, contributing to $50 \%$ of the variance in an ensemble of forecast runs (Yang et al., 2016). Since the dissipation rate quantifies the amount of TKE that is converted into heat at the smallest scales of motion, observations must be localized and highly resolved temporally. Historically, sonic anemometers mounted on towers (Kaimal et al., 1968; Kaimal, 1978), tethered balloons (Frehlich et al., 2003), or aircraft (Nicholls, 1978; Lemone and Pennell, 1980) have provided high-quality in situ observations for this purpose, but towers are rare and aircraft are expensive.

Hocking (1985) showed the feasibility of observing profiles of turbulence in the mesosphere with wind profiling radars (WPRs), which measure backscatter intensity off clear-air parcels in the radar volume during a period of time, which is converted into a Doppler spectrum of velocities. Turbulence intensities (velocity fluctuations divided by the mean velocities) using refractive index and TKE dissipation 
rates using spectral widths of the Doppler velocity spectrum were introduced therein, but Cohn (1995) showed that the TKE dissipation rates were more accurately measured by the WPR than the turbulence intensity. Since then, much progress has been made to improve spectral width measurements for dissipation rate estimations through the removal of non-turbulent broadening effects from wind shear and antennae geometry (Gossard, 1990; Nastrom, 1997; Nastrom and Eaton, 1997; White et al., 1999). The full integration of the method into routine use has not taken place, however, due to unidentified limitations in the method's ability to measure the full range of dissipation rates resolved by highfrequency instrumentation, such as sonic anemometers or hot-wire anemometers (Oncley et al., 1996). To this date, the most state-of-the-art results are found in Shaw and LeMone (2003) and Jacoby-Koaly et al. (2002), studying the PBL. Shaw and LeMone (2003) used a $915 \mathrm{MHz}$ WPR to estimate dissipation rates using spectral widths, and found higher values than the in situ observations, even after the non-turbulent broadening terms were removed from the observed widths (see their Fig. 3), likely due to use of a standard WPR setup with coarse spectral resolution. Jacoby-Koaly et al. (2002) found better agreement between aircraft measurements of dissipation and the ultra-high-frequency $(1238 \mathrm{MHz}) \mathrm{WPR}$ but discussed the inability to accurately remove the effects of the large beamwidth on spectral width. It is worth mentioning that in both of these studies, when analyzed on a linear scale, the overestimations at the smallest dissipation rates are given less significance.

Here, we also use a $915 \mathrm{MHz}$ WPR, set up with higher spectral resolution for more accurate measurements of small spectral widths. Along with several parameters and postprocessing techniques, we investigate the ability of the $915 \mathrm{MHz}$ WPR, as well as a $449 \mathrm{MHz}$ WPR, run nearby to a highly instrumented $300 \mathrm{~m}$ tower, to estimate accurate turbulence dissipation rates throughout the PBL. With these measurements, comparisons can be made on timescales appropriate for evaluation of numerical weather prediction models, $\mathcal{O}$ (1 h).

This paper is organized as follows: Sect. 2 introduces the observations used, including the sonic anemometers and WPRs. Section 3 discusses the methods of calculating dissipation rates from both sets of instruments. Section 4 describes the different post-processing procedures that will be tested for their impact on dissipation rate estimations in Sect. 5 (449 MHz WPR) and Sect. 6 (915 MHz WPR), respectively. Section 7 discusses a useful bias correction. Section 8 describes the uncertainty quantification applied, and Sect. 9 contains the conclusion.

\section{Observations}

All observations in this study were gathered at the Boulder Atmospheric Observatory (BAO), operated by the Na- tional Oceanic and Atmospheric Administration's Earth System Research Laboratory. The site has gently sloping terrain and is located $20 \mathrm{~km}$ east of the foothills of the Front Range of the Rocky Mountains, about $30 \mathrm{~km}$ north of Denver, Colorado. A $300 \mathrm{~m}$ meteorological tower instrumented with sonic anemometers and temperature and humidity sensors is located at the site. During the eXperimental Planetary boundary layer Inter-comparison Assessment (XPIA) in March to June of 2015, the tower was instrumented with pairs of sonic anemometers at six heights up to $300 \mathrm{~m}$. Two WPRs were functioning as well, from 1 March to 30 April 2015, located $600 \mathrm{~m}$ from the tower at the BAO Visitors' Center. A detailed description of the field campaign and all instruments included can be found in Lundquist et al. (2016). Data are available at the Department of Energy's Data Archive and Portal at http://a2e.energy.gov/data\#xpia.

\subsection{Sonic anemometers}

During XPIA, the $300 \mathrm{~m}$ tower at the BAO was equipped with twelve Campbell Scientific CSAT3 sonic anemometers (commonly called sonics), provided by the Characterizing the Atmospheric Boundary Layer (CABL) program of the National Center for Atmospheric Research and the University of Colorado. Sonics were mounted on two booms extending to 154 and $334^{\circ}$ from north, at $50 \mathrm{~m}$ intervals from 50 to $300 \mathrm{~m}$, sampling at $20 \mathrm{~Hz}$. The sonics recorded threedirectional velocities, with $\boldsymbol{u}$ aligned into the boom and $\boldsymbol{v}$ aligned $90^{\circ}$ to the left. The planar fit tilt correction and rotation algorithm of Wilczak et al. (2001) was applied to these data to remove any vertical tilt of the instrument $\left(<2^{\circ}\right.$ for all instruments) and to align the mean velocities into the $30 \mathrm{~min}$ mean wind direction (so that $\overline{\boldsymbol{v}}=0 \mathrm{~m} \mathrm{~s}^{-1}$ ). Data in the wake of the tower were rejected, based on the 1 min mean winds from the upstream boom, from 319 to 344 and from 121 to $197^{\circ}$ (from N), as determined by McCaffrey et al. (2017b). Data were also quality controlled for signal amplitude, signal lock, and the difference in the speed of sound between the three nonorthogonal axes. The tilt-corrected, rotated, qualitycontrolled data from pairs of sonics were then used for calculations of turbulence dissipation rates as detailed in Sect. 3.1 and averaged over $30 \mathrm{~min}$ intervals for comparison to the WPR observations.

\subsection{Wind profiling radars}

Two wind profiling radars, located $600 \mathrm{~m}$ southwest of the BAO tower, are included in the analysis: a $449 \mathrm{MHz}$ radar and a $915 \mathrm{MHz}$. For each hour, the systems ran in standard acquisition mode for $25 \mathrm{~min}$ for three-dimensional consensus winds, in radio acoustic sounding system (RASS) mode for $5 \mathrm{~min}$ for measurements of virtual temperature, and in an optimized turbulence mode (described below) with only a vertically pointed beam for the remaining $30 \mathrm{~min}$. Time series of amplitude and phases (I and Q) were saved dur- 


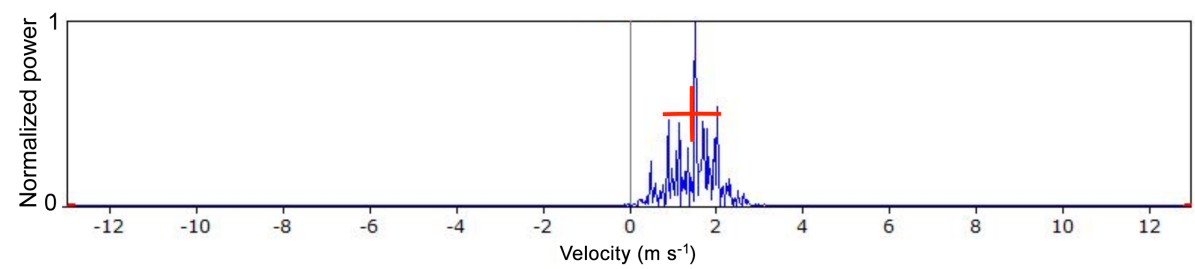

Figure 1. A normalized Doppler spectrum collected from the $499 \mathrm{MHz}$ WPR during the XPIA field campaign with high spectral resolution. The vertical red line denotes the first moment (mean velocity) and the horizontal red line denotes the spectral width, using the standard peak processing method.

ing the $30 \mathrm{~min}$ of turbulence mode to allow the testing of different post-processing settings and methods. The radars measure reflectivity in the radial direction, averaging across a quasi-cylindrical volume with height, $\Delta R$, here equal to $25 \mathrm{~m}$. WPR complex time series were filtered for birds, radio-frequency interference, ground clutter, and other nonatmospheric contamination using wavelet and Gabor postprocessing techniques (Jordan et al., 1997; Lehmann, 2012). Fast Fourier transformations (FFTs) were then computed to obtain Doppler spectra (power vs. velocity), as shown in Fig. 1 from the $449 \mathrm{MHz}$ WPR. The Doppler spectra are retrieved at a temporal resolution, or a dwell time, of $\Delta t$, determined by the radar setup parameters:

$$
\Delta t=[\mathrm{IPP}][\mathrm{NCOH}][\mathrm{NFFT}][\mathrm{NSPEC}],
$$

where IPP is the inter-pulse period (ns), $\mathrm{NCOH}$ is the number of coherent integrations, NFFT is the number of points used in the FFT, and NSPEC is the number of spectral averages. "Optimized" turbulence mode refers to the high spectral resolution, accomplished with a large NFFT, and high temporal resolution (small $\Delta t$ ) due to no spectral averaging $(\mathrm{NSPEC}=1)$. The details of the radar setups are shown in Table 1. If desired, averaging of Doppler spectra can be performed to lower the noise level, thereby increasing the signal-to-noise ratio (SNR) and lengthening the dwell time. A threshold that determines whether a signal is discernible was applied to the spectra based on the SNR. The threshold, developed by Riddle et al. (2012), defines the minimum SNR for detectability to be

$$
\mathrm{SNR}_{\min }=10 \log \left[\frac{25\left(\mathrm{NSPEC}-2.3125+\frac{170}{\mathrm{NFFT}}\right)^{1 / 2}}{\mathrm{NFFT} \times \mathrm{NSPEC}}\right] .
$$

Next, the first and second Doppler spectral moments (mean velocity and spectral width, respectively) are obtained through peak processing algorithms, shown in the red vertical and horizontal lines in Fig. 1. The second moments of the Doppler velocity spectra are then used in the calculation of dissipation rate, as described in Sect. 3.2. The six levels of the sonic anemometers overlapped with six of the $915 \mathrm{MHz}$ profiler's range gates (50 to $300 \mathrm{~m}$ ) and four of the $449 \mathrm{MHz}$ WPR's (150 to $300 \mathrm{~m}$ ).
Table 1. Radar parameters for the 449 and $915 \mathrm{MHz}$ wind profiling radars, running in turbulence mode for minutes 25-55 of each hour during XPIA from 1 March to 30 April 2015.

\begin{tabular}{lrr}
\hline Radar freq $(\mathrm{MHz})$ & 449 & 915 \\
\hline Inter-pulse period $(\mu \mathrm{s})$ & 33 & 45 \\
No. coherent integ. & 24 & 182 \\
NSPEC & 1 & 1 \\
NFFT & 16384 & 2048 \\
First gate height (m) & 154 & 76 \\
No. of range gates & 80 & 72 \\
Range gate height $(\mathrm{m})$ & 26 & 25 \\
Dwell time $(\mathrm{s})$ & 12.98 & 16.77 \\
Spectral resolution $\left(\mathrm{m} \mathrm{s}^{-1}\right)$ & 0.025 & 0.01 \\
\hline
\end{tabular}

\section{Dissipation rate calculations}

When modeling the turbulent atmosphere, the budget of the TKE is needed for parameterizing the small-scale processes that models cannot resolve. The TKE budget can be described as

$$
\frac{\mathrm{d}}{\mathrm{d} t}(\mathrm{TKE})+\nabla \cdot \boldsymbol{T}=P-\varepsilon,
$$

where $\frac{\mathrm{d}}{\mathrm{d} t}$ is the material derivative, $\boldsymbol{T}$ is the turbulent transport, $P$ is the production, and $\varepsilon$ is the dissipation rate. The dissipation rate can be estimated with several methods, for sonic anemometers (Kaimal et al., 1968; Champagne, 1978; Oncley et al., 1996), ultra-high-frequency WPRs (Gossard, 1990; Jacoby-Koaly et al., 2002), and Doppler wind lidars (Smalikho and Banakh, 2013). The foundational work by Kolmogorov (1941) provided a basic dimensional argument for relating the dissipation rate to the transfer of kinetic energy between scales of motions, assuming isotropic, homogeneous, stationary turbulence. In this case, energy is injected at large scales and transferred to small scales, where it dissipates, with no energy lost in the intermediate scales, called the "inertial range". The energy spectral density for each velocity component, $\boldsymbol{i}=[u, v, w]$, can therefore be represented in terms of only the dissipation rate and wave number (scale), $k$ :

$\phi_{E, i}=\alpha_{i} \varepsilon^{2 / 3} k^{-5 / 3}$, 
where $\alpha_{i}$ is a constant (and $\alpha_{v}=\alpha_{w}$ ). The spectrum can be written in terms of frequency, $f$, instead of wave number assuming $k=2 \pi f / \bar{u}$ :

$k \phi_{E, i}(k)=\alpha_{i}\left(\frac{2 \pi}{\bar{u}}\right)^{-5 / 3} \varepsilon^{2 / 3} f^{-5 / 3}$.

The integral of the energy spectrum in the inertial range is equal to the total variance. Assuming this basic form for the energy spectrum, and the relationship to $\varepsilon$, the dissipation rates can be calculated from both the radar profilers and the sonic anemometers, using different methods for each dataset, to close the TKE budget.

\section{1 $\varepsilon$ from sonic anemometer energy spectra}

For all time intervals when $\phi_{E}(f)$ has a $-5 / 3$ power law, the observed power spectral density can be used to calculate the dissipation rate. This can be accomplished using in situ sonic anemometers, which operate at sufficiently high frequencies $(20 \mathrm{~Hz})$ to resolve the inertial range. Using the highsampling-frequency measurements by the sonic anemometers, the dissipation rate can be directly obtained from the energy spectrum. The entire spectrum, however, contains potential errors that must be removed before calculating the dissipation rates. To account for the errors introduced from Taylor's hypothesis, Wyngaard and Clifford (1977) obtained a modification, $T_{i i}$, on the observed spectrum as follows:

$$
\begin{aligned}
& T_{u u}=1-\frac{1}{9} \frac{\sigma_{u}^{2}}{\bar{U}^{2}}+\frac{2}{3} \frac{\sigma_{v}^{2}}{\bar{U}^{2}}+\frac{2}{3} \frac{\sigma_{w}^{2}}{\bar{U}^{2}} \\
& T_{v v}=T_{w w}=1-\frac{1}{9} \frac{\sigma_{u}^{2}}{\bar{U}^{2}}+\frac{2}{3} \frac{\sigma_{v}^{2}}{\bar{U}^{2}}+\frac{1}{3} \frac{\sigma_{w}^{2}}{\bar{U}^{2}}
\end{aligned}
$$

where the subscripts $i=u, v$, and $w$ denote the components of the velocity vector (longitudinal, transverse, and vertical, respectively). Similarly, the averaging along the sonic path introduces an error in the amplitude of the energy spectrum, so Kaimal et al. (1968) developed a spectral transfer function (stf) to reduce this impact:

$T_{\mathrm{stf}}(k d)=\frac{\sin ^{2}(k d / 2)}{(k d / 2)^{2}}$,

where $d$ is the path length, equal to $15 \mathrm{~cm}$ for the sonic anemometers used here. The modified energy spectrum is then defined as

$\phi_{\text {new }}(k)=\phi_{E, i}(k) \cdot T_{\mathrm{stf}}(k d) \cdot T_{i i}$.

Figure 2 shows one 15 min velocity spectrum for the horizontal (blue) and vertical (red) velocities with (bright colors) and without (pale colors) the adjustments. The adjustment is small but used for thoroughness, since it extends the inertial range. Equation (9) can be inverted and averaged over the inertial range (here, identified by visual inspection to be from

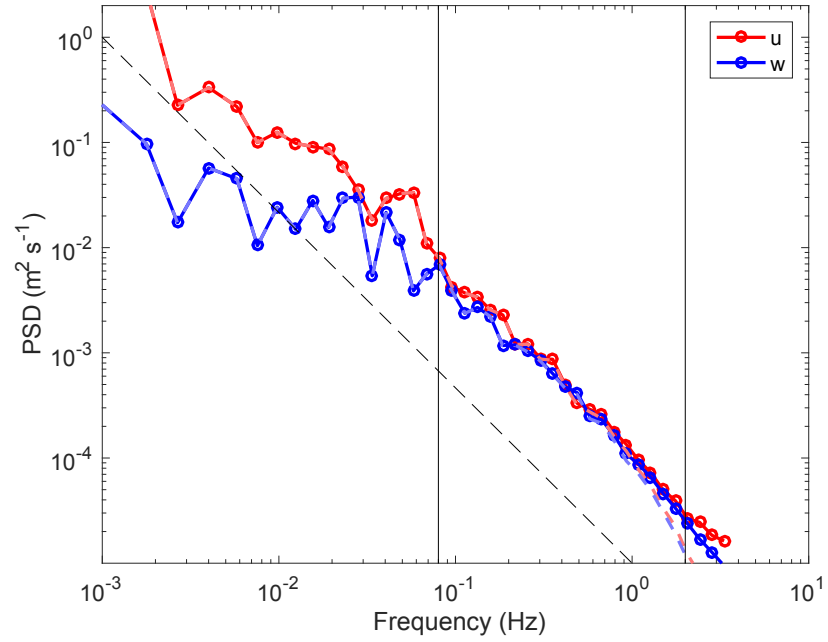

Figure 2. Velocity spectra for the horizontal (blue) and vertical (red) velocities with (bright colors with circles) and without (pale colors, dashed lines) the adjustments, for the northwest sonic anemometer at $150 \mathrm{~m}$ on the BAO tower. A spectral slope of $-5 / 3$ is shown as the dashed line for reference, and vertical lines denote the range used for integration.

$8 \times 10^{-2}$ to $2 \times 10^{0} \mathrm{~s}^{-1}$, as denoted by the solid vertical lines in Fig. 2), as in Kaimal et al. (1968), to solve for $\varepsilon$ :

$\varepsilon=\frac{1}{\alpha_{u}^{3 / 2}}\left(\frac{2 \pi}{\bar{u}}\right)^{3 / 2}\left\langle\left[f^{5 / 3} \phi_{\text {new }}(f)\right]^{3 / 2}\right\rangle$,

where the angled brackets are averages in frequency. Energy spectra were computed for each 15 min interval, and corresponding dissipation rates were calculated in those which were verified to have a slope of $-5 / 3$ in the inertial range. This verification process included an automated calculation of the slope of the best fit line in this range, and slopes within a range from $-4 / 3$ to $-6 / 3$ were used. Since this method relies on assumptions about the energy spectrum, dissipation rates from sonic anemometers are still only estimations of the true values (as observed by hot-wire anemometers: Tennekes and Lumley, 1972), but since Kaimal et al. (1968) they have been used as the true measurement. A quantification of the uncertainty in the sonic anemometers' dissipation rates based on the variance of the energy spectrum in the inertial range will be introduced in Sect. 8. The dissipation rates were then averaged over $30 \mathrm{~min}$ to compare with those obtained from the WPRs.

\section{2 $\varepsilon$ from radar spectral widths}

The method of calculating dissipation rates from WPRs uses the width of the radar Doppler spectrum (spectral width) rather than the velocity energy spectrum, like the sonic anemometers' method, since the time resolution of the velocity moments is insufficient to resolve an inertial range. The spectral width method is also applied to sodars (Ottersten 
et al., 1973) and Doppler lidar (Smalikho and Banakh, 2013) spectra for dissipation rates, though the structure function and velocity azimuth display methods are more accurate and common (Sathe and Mann, 2013). The radar Doppler spectrum provides the distribution of velocities of backscattering particles within the radar volume, with an average equal to the mean velocity and a spread which indicates the level of turbulence. The spectrum measured by a profiling radar contains both the resolved velocities in the mean wind speed, and the unresolved velocities in the spectral width (Angevine et al., 1994; McCaffrey et al., 2017a). The spectral width of the Doppler spectrum is defined to be twice the standard deviation, $\sigma_{\mathrm{m}}$, of the unresolved velocities in the measurement volume during each dwell. This total measured variance, $\sigma_{\mathrm{m}}^{2}$, includes the effects of turbulence, as well as non-turbulent effects such as wind shear and antenna effects. The total variance is made of independent contributors, so it can be summarized as follows:

$\sigma_{\mathrm{m}}^{2}=\sigma_{\mathrm{s}}^{2}+\sigma_{\mathrm{t}}^{2}$,

where $\sigma_{\mathrm{s}}^{2}$ is the variance due to wind shear and beambroadening effects, and $\sigma_{\mathrm{t}}^{2}$ is due to turbulence (Gossard, 1990). Nastrom (1997) has determined the shear and beambroadening term to depend on the mean wind transverse to the beam axis, $V_{\mathrm{T}}$, the mean wind shear, $\mathrm{d} \bar{u} / \mathrm{d} z$, and the antenna properties. For a vertically pointing beam, the contribution is determined to be

$\sigma_{\mathrm{s}}^{2}=\frac{v^{2}}{3}\left(V_{\mathrm{T}}^{2}+\left(\frac{\mathrm{d} \bar{u}}{\mathrm{~d} z}\right)^{2} \frac{\Delta R^{2}}{12}\right)$,

where $v$ is the half-width to the half-power point in the antenna pattern and $\Delta R$ is the range gate. Nastrom (1997) also determined the broadening contribution due to gravity waves across the radar beam, but for a single, vertically pointing beam the contribution is small and therefore neglected. In some cases, however, the contribution to the spectral width from the shear and beam-broadening term, $\sigma_{\mathrm{s}}^{2}$, is larger than the total measured width, $\sigma_{\mathrm{m}}^{2}$, creating negative values of dissipation rates. These are not physically meaningful, so these values are rejected. Despite possibly skewing the $30 \mathrm{~min}$ averages by neglecting values which may be small (Dehghan et al., 2014), comparisons showed that all other solutions (e.g., substituting with 0 or a small value of $\sigma$, or not removing any broadening) produced unphysical or statistically worse results. Figure 3 shows the percentage of 2 min dwells $(\mathrm{NSPEC}=8)$ that experience $\sigma_{\mathrm{s}}^{2}>\sigma_{\mathrm{m}}^{2}$. The $915 \mathrm{MHz}$ WPR experienced this up to $40 \%$ of the time, though the fraction decreases with more spectral averages, as the measured widths are broadened with longer dwell times (and the beam broadening remains constant). The $449 \mathrm{MHz}$ WPR, with a narrower beam and higher SNR, is not as impacted by this issue, except at the lowest range gate, where ground clutter causes noisier spectra.

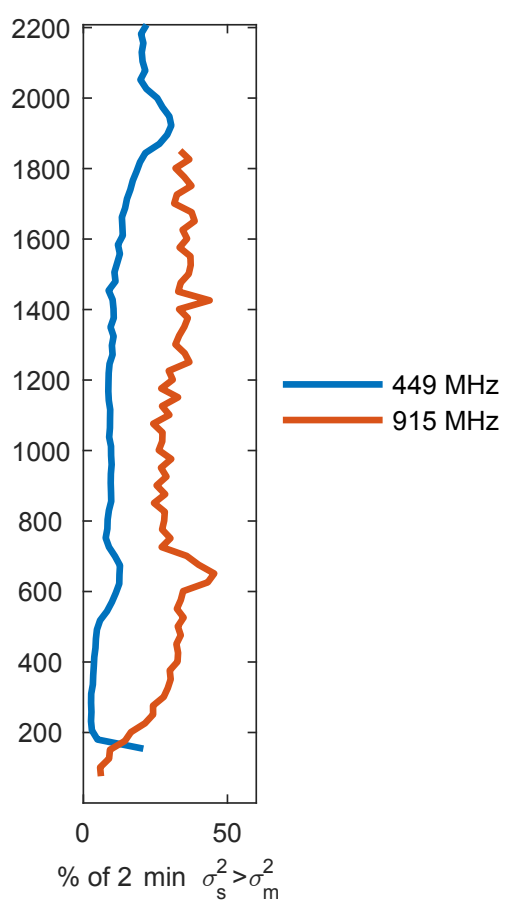

Figure 3. Percentage of 2 min dwells $(\mathrm{NSPEC}=8)$ when $\sigma_{\mathrm{s}}^{2}>\sigma_{\mathrm{m}}^{2}$ for the 449 (blue) and $915 \mathrm{MHz}$ (red) WPR, using the standard peak processing method of calculating $\sigma_{\mathrm{m}}^{2}$.

The work by Frisch and Clifford (1974), Labbitt (1981), and Gossard et al. (1998) led White et al. (1999) to an expression for the relationship between the contribution to the spectral width due to turbulence and the dissipation rate, in a spectral form:

$$
\begin{aligned}
& \sigma_{\mathrm{t}}^{2}=\frac{\alpha \varepsilon^{2 / 3}}{4 \pi} \iiint k^{-11 / 3}\left[1-\left(\frac{k_{1}}{k}\right)^{2}\right] \\
& {\left[1-\frac{\sin ^{2}\left(k_{2} L / 2\right)}{\left(k_{2} L / 2\right)^{2}} \exp \left[-b^{2} k_{1}^{2}-a^{2}\left(k_{2}^{2}+k_{3}^{2}\right)\right]\right] \mathrm{d} k_{1} \mathrm{~d} k_{2} \mathrm{~d} k_{3},}
\end{aligned}
$$

where $\alpha=1.6$ is a Kolmogorov constant, $L=V_{\mathrm{T}} \Delta t, a$ is the half-diameter of the (circular) beam cross section, $b$ is the half-length of the pulse, and $k_{i}$ are the three components of the wave number. The integral in Eq. (13) can be solved by converting to spherical coordinates:

$$
\begin{aligned}
I= & 12 \Gamma\left(\frac{2}{3}\right) \int_{0}^{\frac{\pi}{2}} \mathrm{~d} \phi \int_{0}^{\frac{\pi}{2}} \mathrm{~d} \theta \sin ^{3} \theta \\
& \left(b^{2} \cos ^{2} \theta+a^{2} \sin ^{2} \theta+\frac{L}{12} \sin ^{2} \theta \cos ^{2} \phi\right)^{1 / 3},
\end{aligned}
$$

where $\Gamma$ is the gamma function. This equation can be numerically integrated, and Eq. (13) can be solved for $\varepsilon$.

The dissipation rates were estimated for the $30 \mathrm{~min}$ of turbulence mode, during which the time series of amplitudes and phases (I and Q) were saved, and different 
post-processing and moment-calculation methods were performed. A quantification of the uncertainty in the WPRs' dissipation rates will be introduced in Sect. 8 .

\section{Post-processing procedures}

During the calculation process for spectral moments from WPRs, there are several options and parameters to be considered that have the possibility of improving the accuracy of the spectral width measurements. These options include radar setup, time series filtering (of amplitude and phase signal), Doppler spectral processing, and moment calculations, all of which have an effect on the final spectral width used for dissipation rates. Here we will investigate the effect of standard and multiple peak processing methods and noise level thresholds of moment calculations, and spectral averaging on the eddy dissipation rate as determined with the WPRs' spectral width, using the in situ observations from sonic anemometers for comparison.

\subsection{Moment calculations: standard vs. multiple peak processing}

Once the Doppler spectra are calculated (with wavelet and Gabor filtering applied) and processed for ground clutter (Riddle and Angevine, 1991) and other interference reduction, the first two moments of the Doppler spectra are calculated by one of two common methods. The first is the standard peak processing method (SPP) using the basic method of finding the highest Doppler peak at each range gate, then integrating between the velocities, $v_{1}$ and $v_{2}$, where the noise level (maximum or mean) is surpassed. The second method of calculating the moments, the multiple peak processing method (MPP) finds up to three peaks at each range gate with the highest power, then uses continuity in time and space (vertical profiles) to determine which peak is most likely to be the true signal. The velocity range of the peak is determined by the crossing of the mean noise level or an increase in power due to an adjacent peak. The velocity limits, $v_{1}$ and $\nu_{2}$, of the chosen peak then go into the calculation of first and second moments for velocity and spectral width. MPP is the preferred method for the measurement of first-moment winds, as determined in Gaffard et al. (2006), but the impact on the width has not been studied.

When using SPP, the noise level threshold that determines the velocity limits in the calculation of the spectral width can be set to either the maximum noise level of the spectrum (SPP max) or the mean noise level (SPP mean). The common choice is the maximum noise level since it is the most conservative for removing noise and produces a more accurate first moment of the spectrum. However, a non-atmospheric signal could create an artificially high maximum noise level, causing the spectral width to be narrowed. The mean noise level in these cases would be more representative of the true noise in the spectrum and potentially allows the measured spectral widths to be more realistic. A comparison of dissipation rates using these three methods (SPP max, SPP mean, and MPP) with the in situ observations from the sonic anemometers will indicate which moment calculation method is most accurate for measuring spectral widths and, consequently, dissipation rates. All other variables in the calculation of dissipation rate are equal across different moment calculation methods, so the accuracy of dissipation rates indicates the accuracy of the spectral width measurements in each method.

\subsection{Spectral averaging}

Each dwell collected by the $449 \mathrm{MHz}$ WPR spans about $13 \mathrm{~s}$ (and the $915 \mathrm{MHz}$, about $17 \mathrm{~s}$; see Table 1), capturing only a short period of the atmosphere's motions. Therefore, turbulence observed in that dwell time does not completely capture the full characteristics of the flow. More commonly, atmospheric turbulence statistics, such as turbulence intensity or turbulent kinetic energy, are calculated using 2 to $30 \mathrm{~min}$ averages of fluctuations to include a more complete range of scales of turbulence. In the case of Doppler spectra from predetermined radar pulses, spectra from multiple dwells can be averaged together to span a longer time period of fluctuations, resulting in more representative turbulence statistics. Furthermore, spectral averaging decreases the noise in the resulting spectra, allowing for cleaner atmospheric peaks. However, averaging over periods that are too long, and therefore nonstationary, will result in broadening of the spectral peak that is due to a shifting mean velocity rather than due to true fluctuations from turbulence. McCaffrey et al. (2017a) analyzed the impact of lengthened averaging times on vertical velocity variance measurements and found that the optimal time for spectral width calculations was $2 \mathrm{~min}$. Here, an analysis was performed to determine the length of time, set by the number of spectral averages, which produces the most accurate dissipation rates compared to the in situ observations from the sonics.

\section{Comparison of $\varepsilon$ from $449 \mathrm{MHz}$ WPR and sonic anemometers}

With the use of the in situ sonic anemometer observations as the baseline, the different post-processing techniques presented in Sect. 4.1 and 4.2 can be analyzed for their impacts on the resulting dissipation rates. Figure 4 summarizes the behavior of comparisons between the $449 \mathrm{MHz}$ WPR and the sonic anemometers at their four overlapping heights, using MPP (blue) and SPP, with maximum (red) and mean (green) noise levels, as a function of the different numbers of spectral averages. With the un-averaged spectra having a dwell time of $13 \mathrm{~s}$, using NSPEC $=42$ produces dwells of approximately $10 \mathrm{~min}$. In the correlation (measured as $R^{2}$ of the logarithm of dissipation rates; Fig. 4a), mean abso- 

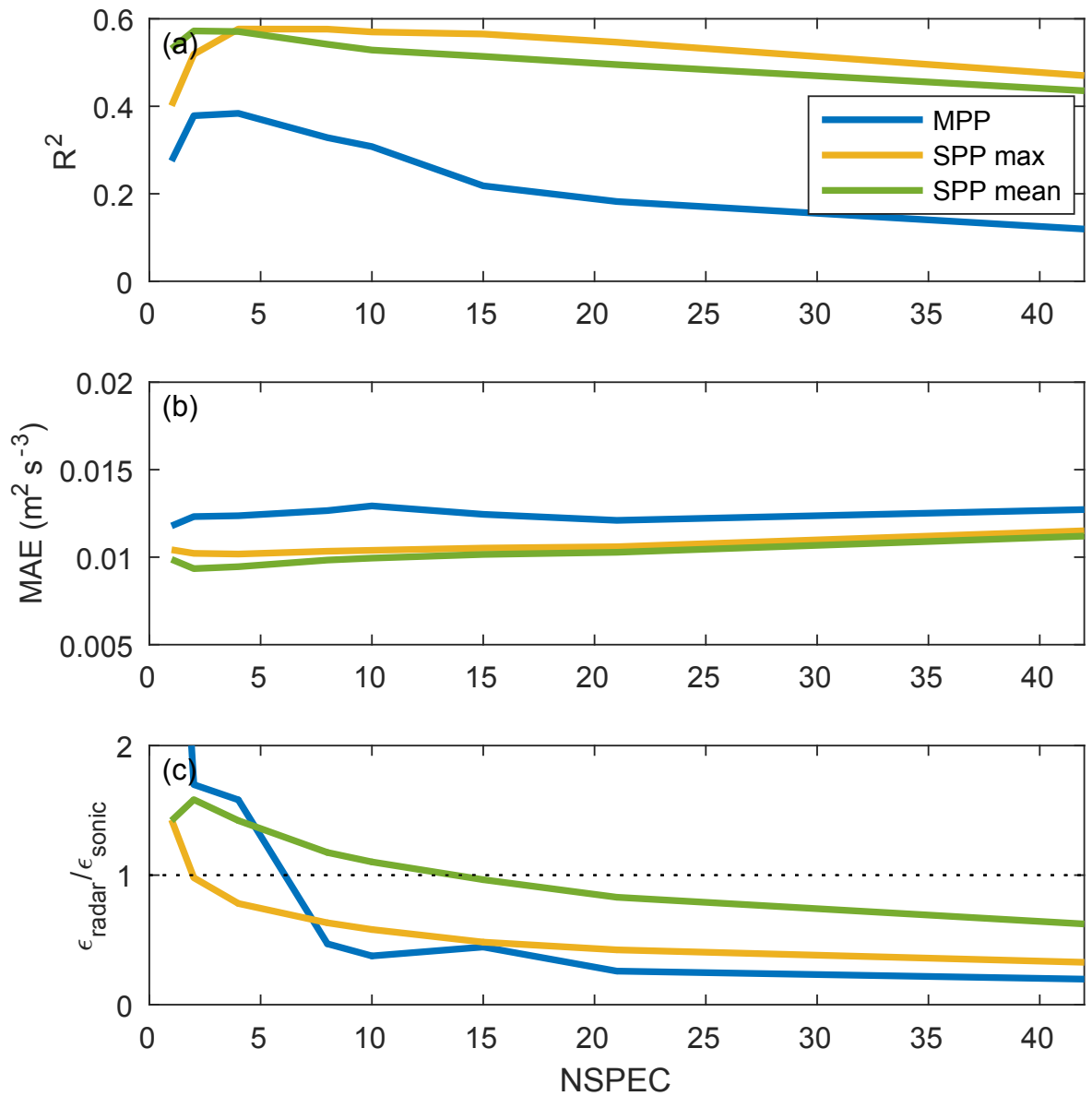

Figure 4. (a) Coefficient of determination, $R^{2}$, of the logarithm of the dissipation rates and (b) mean absolute error, MAE, and (c) fractional bias $\left(\varepsilon_{\text {radar }}\right.$ divided by $\left.\varepsilon_{\text {sonic }}\right)$ of dissipation rate between the sonic anemometers and the $449 \mathrm{MHz}$ using MPP (blue) and SPP with the maximum (yellow) and mean (green) noise level thresholds, as a function of the numbers of spectral averages, NSPEC.

lute error (MAE; Fig. 4b), and fractional bias (Fig. 4c), MPP does significantly worse than both settings of SPP. A scatter plot of dissipation rates from MPP and the sonic anemometer (Fig. 5) shows that, even at the optimal averaging time $(\mathrm{NSPEC}=4)$ as determined by Fig. 4, MPP often highly underestimates the spectral width that contributes to the dissipation rate. At this high spectral resolution, the MPP method separates broad atmospheric peaks into multiple narrower peaks and so computes artificially narrow widths within the true, wider peak. Thus, we conclude that MPP should not be used for calculations of spectral widths, particularly at high spectral resolution.

When using SPP with either the maximum or mean noise level, averaging over more than one dwell is immediately an improvement over the shortest dwell times (Fig. 4). The highest correlation of the logarithm of dissipation rates, $R^{2}=0.57$, occurs with the maximum noise level using $\mathrm{NSPEC}=4$, or about 1 min dwells, and remains nearly constant until NSPEC $=15$ (Fig. 4a). The mean noise level sees its highest correlation, also of $R^{2}=0.57$, at $\mathrm{NSPEC}=2$

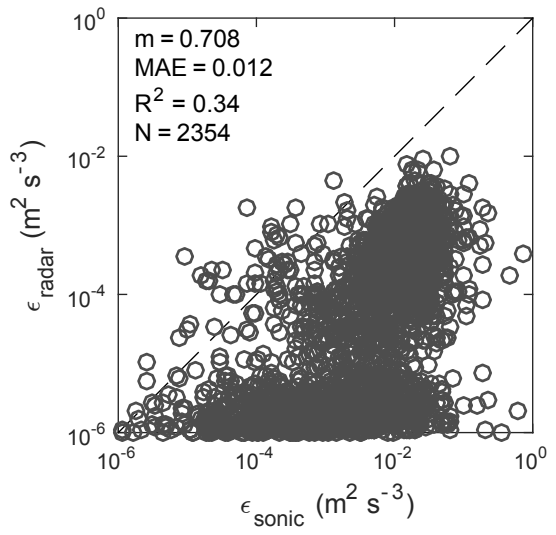

Figure 5. Dissipation rates from the sonic anemometer, $\varepsilon_{\text {sonic }}$, and WPR spectral widths ( $\left.\varepsilon_{\text {radar }}\right)$ from the $449 \mathrm{MHz}$ WPR, using NSPEC $=4$ and MPP to calculate the spectral moments, with slope $(m)$, MAE, $R^{2}$ of the logarithms of dissipation rates, and number of points plotted $(N)$ labeled. 

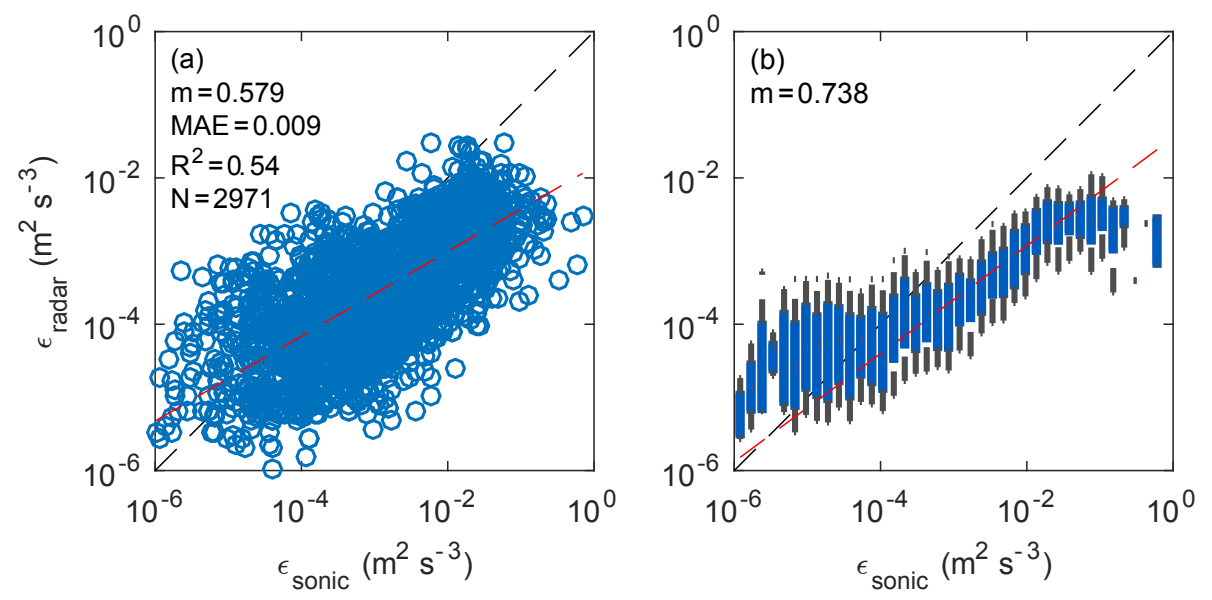

Figure 6. Dissipation rates from the sonic anemometer, $\varepsilon_{\text {sonic }}$, and WPR spectral widths, $\varepsilon_{\text {radar }}$, from the 449 MHz WPR, using SPP with the mean noise level threshold, and NSPEC $=8$, shown in a scatter plot (a), with slope $(m)$, MAE, $R^{2}$ of the logarithms of dissipation rate, and number of points plotted $(N)$ labeled, and as box and whiskers (b), with the blue boxes extending to the 25th and 75th percentiles, black lines to the 1st and 99th percentiles, and central lines at the median. The red dashed lines denotes the best fit line to all points in (a) and the medians of each bin between $\varepsilon_{\text {sonic }}=10^{-4}$ and $10^{-1} \mathrm{~m}^{2} \mathrm{~s}^{-3}$ in (b). The one-to-one line is shown in black dashes on both panels.

(about $\Delta t=30 \mathrm{~s}$ ), then decreases for longer dwell times. The lowest MAE occurs for both noise thresholds at NSPEC $=2$ and increases slightly for longer dwells (Fig. 4b). The MAE is lowest at all NSPEC when using the mean noise level. The bias in SPP is lower using the mean noise threshold at longer timescales, with a minimum (fractional bias equal to 1 ) at $\mathrm{NSPEC}=2$ for the maximum noise level and $\mathrm{NSPEC}=15$ for the mean noise level (Fig. 4c). Optimization of the mean vs. maximum noise level is flexible, through the correlation, MAE, and bias. Similar analysis of the vertical velocity variance, as measured by the WPR spectral widths in McCaffrey et al. (2017a), showed the optimal results at NSPEC $=8$ using the mean noise level in SPP, and that choice for dissipation rates would also give a near-optimal fractional bias with small MAE and high correlation, so future analysis herein will use NSPEC $=8$ and SPP with the mean noise level.

Scatter plots comparing dissipation rates from the sonic anemometers and WPRs using the optimized post-processing procedures are shown in Fig. 6, both as a scatter plot of all $30 \mathrm{~min}$ averages (panel a) and as box-and-whisker plots to more easily see the distribution of values (panel $b$ ). The scatter plot shows a trend near to 1 for larger sonic anemometer values of dissipation (larger than $10^{-4} \mathrm{~m}^{2} \mathrm{~s}^{-3}$ ), but with a near-constant offset from the one-to-one line. The scatter at lower sonic-observed values increases, with more radar values that are overestimated, causing the overall slope of the best-fit line to be much less than 1 . The shift in behavior is more visible on the box plot (Fig. 6b), where the distribution of values departs from the trend near $\varepsilon_{\text {sonic }}=10^{-4} \mathrm{~m}^{2} \mathrm{~s}^{-3}$ and flattens. At the largest values, the box plot also shows a flattening, but the scatter plot shows that there are few points in this range, so the departure from the trend above $\varepsilon_{\text {sonic }}=$ $10^{-1} \mathrm{~m}^{2} \mathrm{~s}^{-3}$ is less significant. Between $\varepsilon_{\text {sonic }}=10^{-4}$ and
$10^{-1} \mathrm{~m}^{2} \mathrm{~s}^{-3}$, the slope of the line fit through the median of each bin (red dashed line in Fig. 6b) has a slope much closer to 1 , with the overall low bias seen as well.

\section{Comparison of $\varepsilon$ from $915 \mathrm{MHz}$ WPR and sonic anemometers}

The $915 \mathrm{MHz}$ WPR operating during XPIA was set up to have similar temporal and spectral resolution as the $449 \mathrm{MHz}$, but the different systems produce spectra with different noise levels and slightly different resolutions (see Table 1). The optimization of dissipation rates through the post-processing techniques of spectral averaging and moment calculations performed for the $449 \mathrm{MHz}$ WPR must be completed separately for the $915 \mathrm{MHz}$ WPR. Figure 7 compares the coefficient of determination, $R^{2}$, MAE, and fractional bias between the $915 \mathrm{MHz}$ WPR and all six overlapping heights of sonic anemometers as a function of the number of spectral averages. The correlations are lower, with higher biases overall, for the $915 \mathrm{MHz}$ WPR than the $449 \mathrm{MHz}$ system. Again, MPP is significantly worse at calculating the spectral width (and, therefore, dissipation rates). There is less of a difference between the noise level thresholds for the $915 \mathrm{MHz}$ WPR than the $449 \mathrm{MHz}$, so for consistency, the mean noise level will be used for further analysis. NSPEC $=8$ shows the best results in terms of all three quantities presented in Fig. 7, showing that a timescale of approximately $2 \mathrm{~min}$ is optimal for measuring spectral widths, with $R^{2}=0.41, \mathrm{MAE}=0.009 \mathrm{~m}^{2} \mathrm{~s}^{-3}$, and fractional bias equal to 1.46 . Figure 8 uses these settings for computation of dissipation rates and is presented as a scatter plot (panel a) and box-and-whisker plot (panel b), with the median and 25th percentiles for each bin of sonic anemometer values. The 

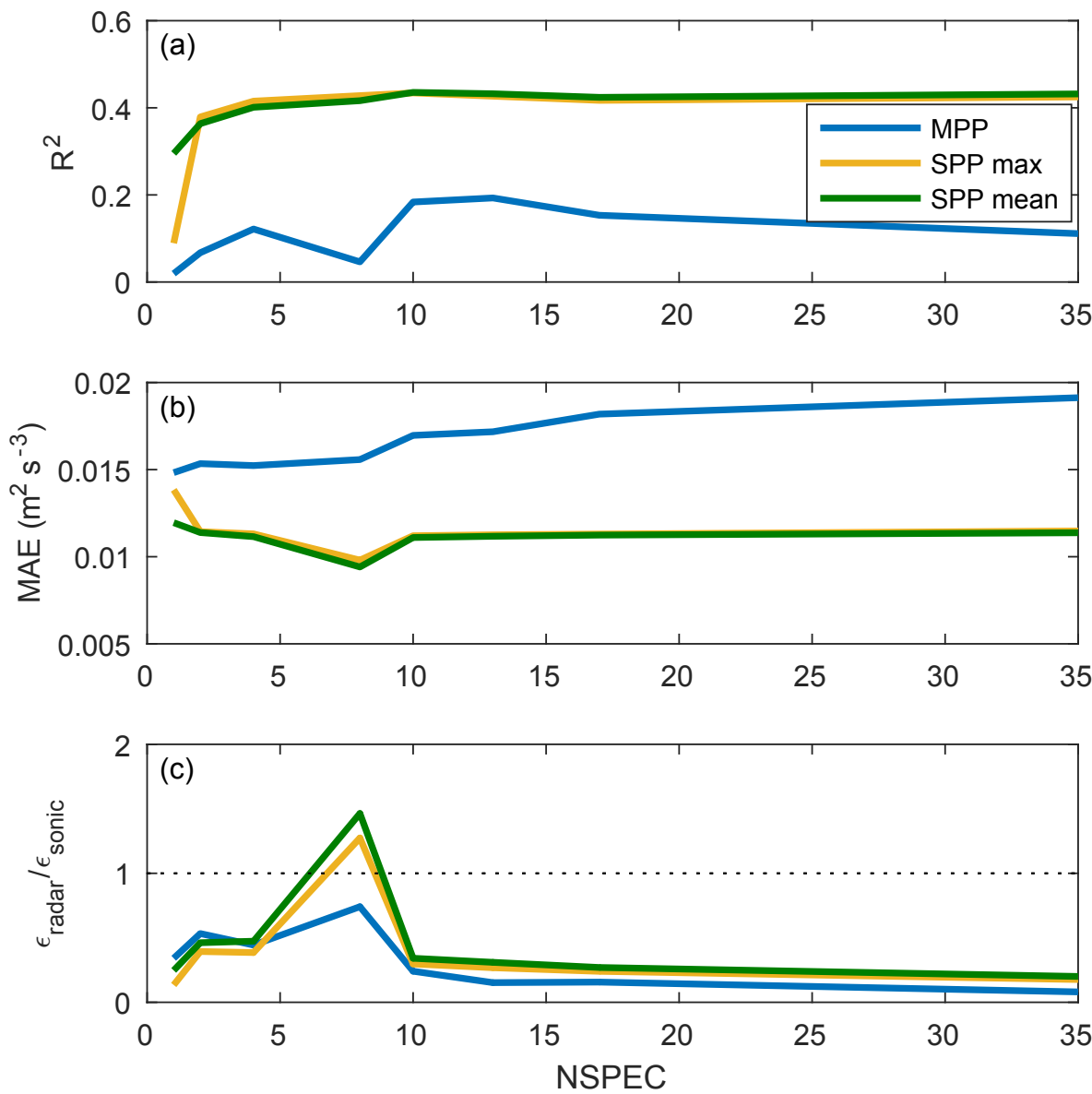

Figure 7. Same as Fig. 4 but for the $915 \mathrm{MHz}$ WPR.
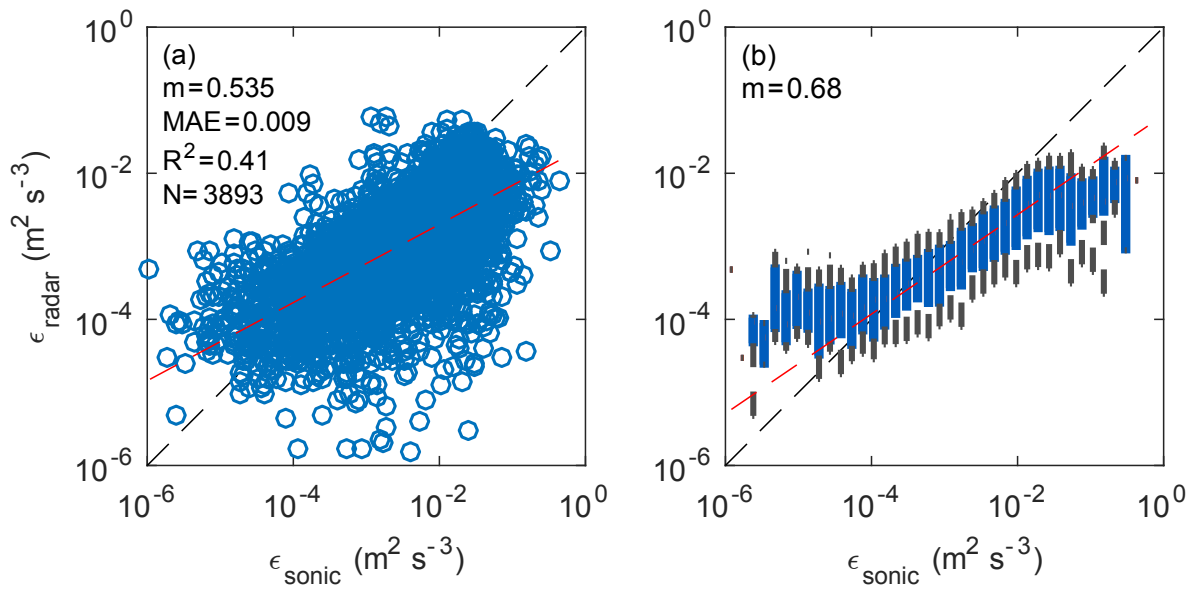

Figure 8. Same as Fig. 6 but for the $915 \mathrm{MHz}$ WPR with NSPEC $=8$ and SPP with the mean noise level. 

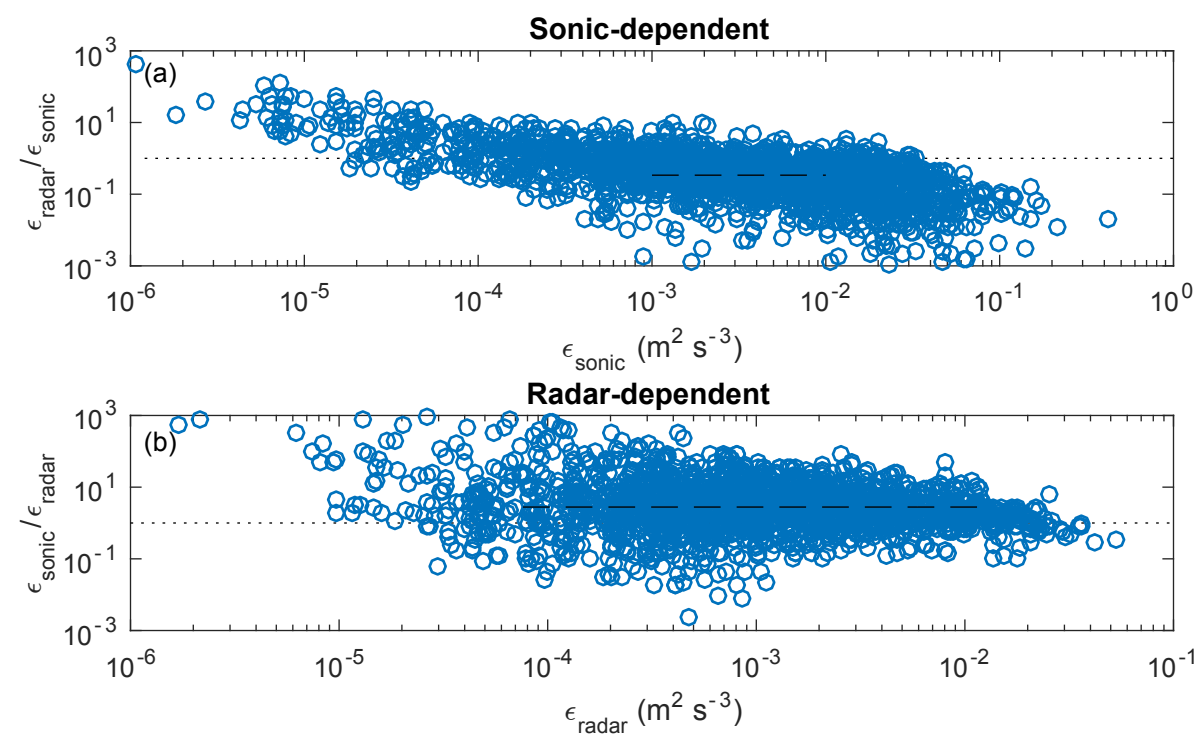

Figure 9. Fractional biases in dissipation rate from all 6 heights of the $915 \mathrm{MHz}$ WPR: $\varepsilon_{\text {radar }} / \varepsilon_{\text {sonic }}$ vs. $\varepsilon_{\text {sonic }}$ (a) and $\varepsilon_{\text {sonic }} / \varepsilon_{\text {radar }}$ vs. $\varepsilon_{\text {radar }}(\mathbf{b})$. Black dashed lines are the averages used in the bias corrections in Table 2, and dashed line denotes a bias of 1 .

overall low bias is again evident, and a trend close to 1 is again seen above $10^{-4} \mathrm{~m}^{2} \mathrm{~s}^{-3}$.

\section{Bias corrections}

The overall biases in dissipation rates seen in Figs. 6a and $8 \mathrm{a}$, and the constant offset in the range $\varepsilon_{\text {sonic }}=10^{-4}$ and $10^{-1} \mathrm{~m}^{2} \mathrm{~s}^{-3}$ in Figs. $6 \mathrm{~b}$ and $8 \mathrm{~b}$ suggest that a bias correction could produce more accurate agreement with the sonic anemometers. A bias correction that is a function of the sonic anemometers values cannot be applied since those values, in most cases, will not be available. Using a constant determined by these WPR-sonic comparisons allows a correction to be performed on other datasets without a priori knowledge of the true value. Two different methods of correcting the observed bias were tested: one corrects based on a constant determined by the dependence on the sonic anemometers, and one is dependent on the observed radar value. The methods are defined in Table 2 with their respective corrections using a function, $\hat{F}(\varepsilon)$, that is averaged in each case. The biases were calculated separately for the two WPRs for the first month of observations (March), reserving the second month (April) for testing the corrections.

Figure 9 presents the fractional biases in the $915 \mathrm{MHz}$ WPR during March as a function of the sonic anemometers $\left(\varepsilon_{\text {radar }} / \varepsilon_{\text {sonic }}\right.$, Fig. 9a) and the WPR ( $\varepsilon_{\text {sonic }} / \varepsilon_{\text {radar }}$, Fig. 9b). The correction based on the sonic-dependent bias (Fig. 9a) must be a constant in order to be applied to other datasets and, therefore, an average in the densest part of the range was chosen (from $10^{-3}$ to $10^{-2} \mathrm{~m}^{2} \mathrm{~s}^{-3}$ ) and is shown as the dashed line. This constant, $c_{1}=0.339$, can then be used to get a corrected value of $\hat{\varepsilon}_{\text {radar }}$ as shown in Table 2 .
Table 2. The two bias correction methods, with their definitions and equations of corrections to the observed $\varepsilon_{\text {radar values. }}$

\begin{tabular}{lll}
\hline & Sonic-dependent & Radar-dependent \\
\hline \multirow{2}{*}{ Definition } & $\frac{\varepsilon_{\text {radar }}}{\varepsilon_{\text {sonic }}}=f\left(\varepsilon_{\text {sonic }}\right) \approx c_{1}$ & $\frac{\varepsilon_{\text {sonic }}}{\varepsilon_{\text {radar }}}=h\left(\varepsilon_{\text {radar }}\right) \approx c_{2}$ \\
\hline \multirow{2}{*}{ Correction } & $\hat{\varepsilon}_{\text {radar }}=\frac{\varepsilon_{\text {radar }}}{c_{1}}$ & $\hat{\varepsilon}_{\text {radar }}=c_{2} \varepsilon_{\text {radar }}$ \\
\hline
\end{tabular}

The second method uses a bias correction dependent on the WPR values, rather than the sonic, as in the first method. The WPR-dependent bias (Fig. 9b) has constant behavior over most dissipation values, so the average between $10^{-4}$ and $10^{-2} \mathrm{~m}^{2} \mathrm{~s}^{-3}$ was used (dashed line). Again, the biascorrection factor, $c_{2}=2.784$, is multiplied by each $\varepsilon_{\text {radar }}$ value to get the corrected $\hat{\varepsilon}_{\text {radar }}$.

To determine the dissipation rates that are most impacted by the bias corrections, Fig. 10 shows the biases in the $915 \mathrm{MHz}$ WPR during March before corrections (blue line) and after correcting, using the sonic-dependent (yellow) and radar-dependent (green) corrections. The observations were binned by the instrument on the dependent axes $\left(\varepsilon_{\text {sonic }}\right.$ in Fig. 10a and $\varepsilon_{\text {radar }}$ in Fig. 10b), and averaged, and the fractional bias was calculated for each bin. In Fig. 10a, the ability of the WPR to estimate each sonic value of $\varepsilon$ is indicated by the biases, highlighting where the radar over- and underestimates the dissipation rate. The improvements can be seen above $\varepsilon_{\text {sonic }}=6 \times 10^{-4} \mathrm{~m}^{2} \mathrm{~s}^{-3}$, where the biases in both corrected datasets (yellow and green lines, which are indecipherably close) are closer to the $100 \%$ line than the original (blue line). However, below $\varepsilon_{\text {sonic }}=6 \times 10^{-4} \mathrm{~m}^{2} \mathrm{~s}^{-3}$, the constant multiplicative adjustment acts in the wrong direc- 

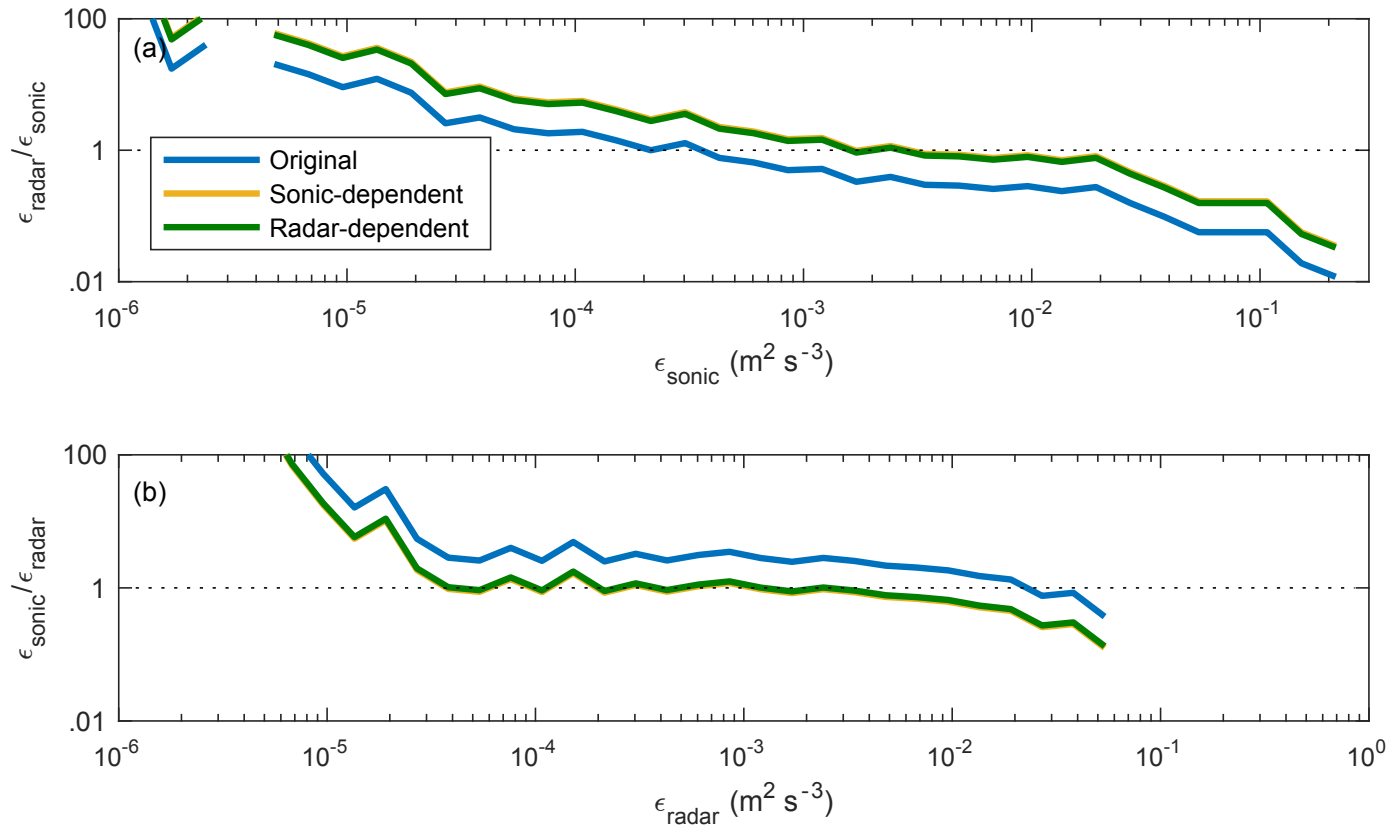

Figure 10. Fractional bias in dissipation rate, defined as (a) $\varepsilon_{\text {radar }} / \varepsilon_{\text {sonic }}$ vs. $\varepsilon_{\text {sonic }}$ and (b) $\varepsilon_{\text {sonic }} / \varepsilon_{\text {radar }}$ vs. $\varepsilon_{\text {radar }}$, during March 2015 from the $915 \mathrm{MHz}$ WPR with no corrections (blue), the sonic-dependent correction (yellow), and radar-dependent correction (green).
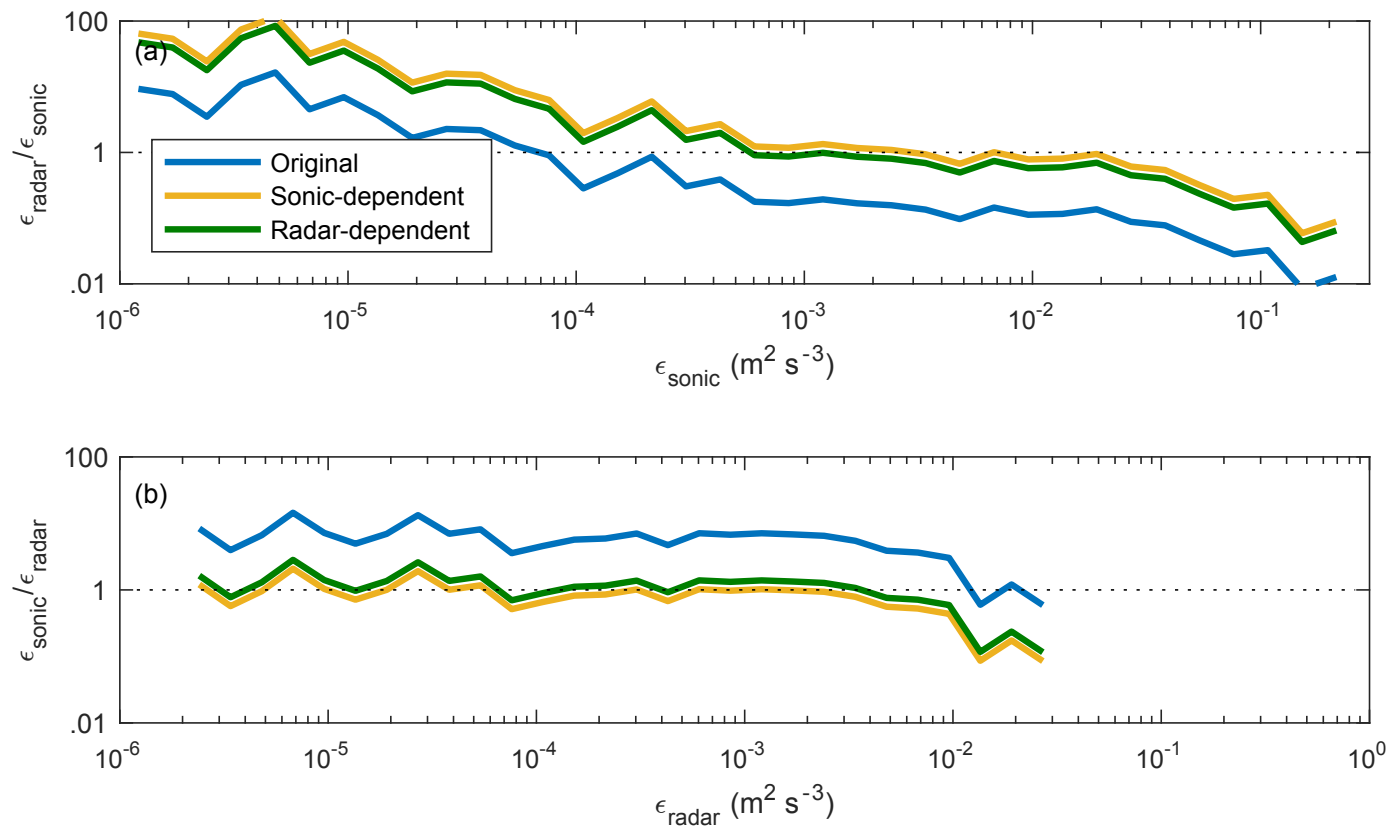

Figure 11. Same as Fig. 10 but for the $449 \mathrm{MHz}$ WPR.

tion, and dissipation rates that were originally overestimated by the WPR are further overestimated.

Analyzing the average biases as a function of the WPRestimated dissipation rate gives insight into the accuracy of those measurements (Fig. 10b). The original dataset always lies above the $100 \%$ line (blue line), indicating that the true sonic value is nearly always higher than the observed WPR values, except at the highest values (which are few, as seen in Fig. 9b). The corrections based on both the radar and the sonic anemometers nearly remove the entire bias for dissipation rates in the range between $\varepsilon_{\text {radar }}=2 \times 10^{-5}$ and $10^{-2} \mathrm{~m}^{2} \mathrm{~s}^{-3}$ and improve all measurements below $\varepsilon_{\text {radar }}=$ $10^{-2} \mathrm{~m}^{2} \mathrm{~s}^{-3}$. 

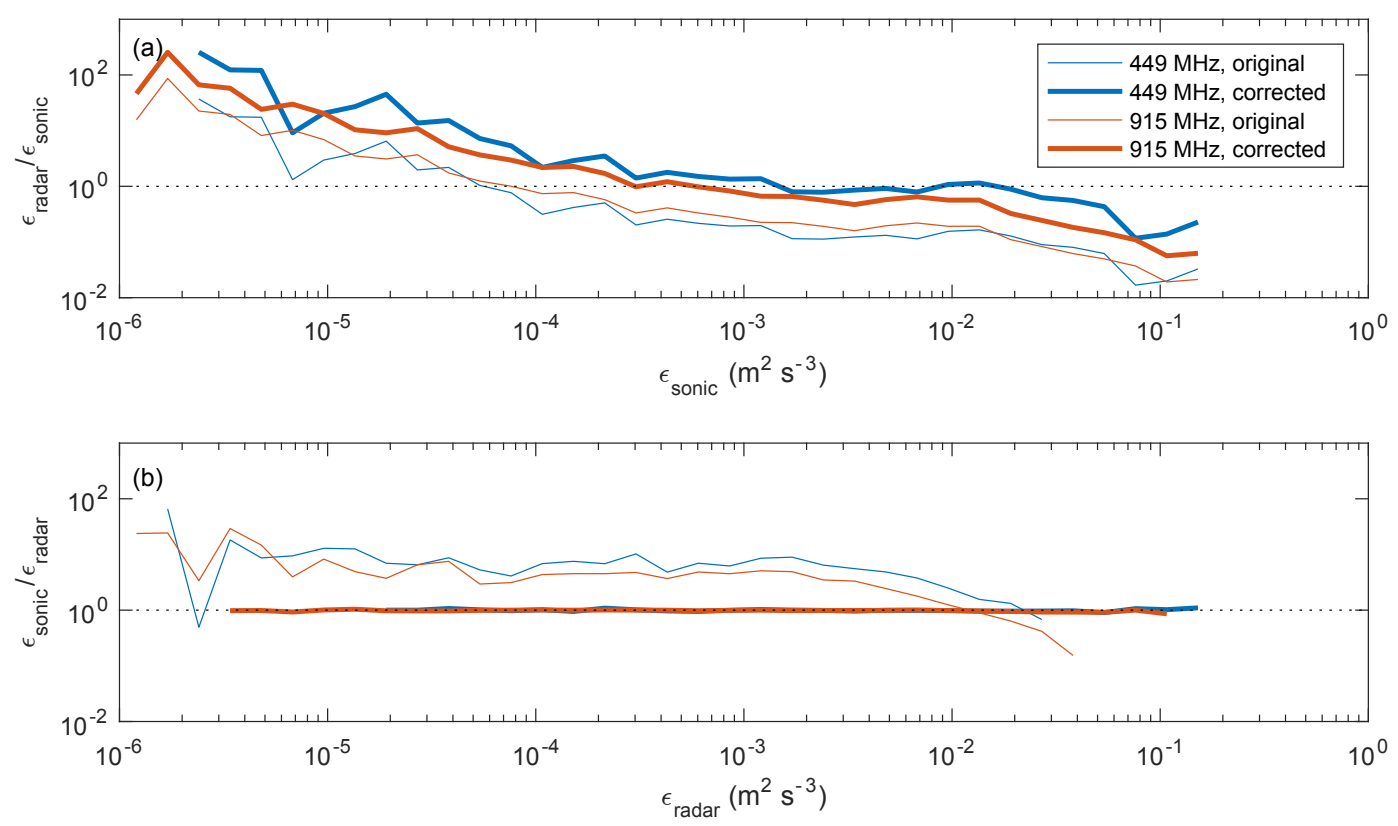

Figure 12. Fractional bias in dissipation rate (defined as $\varepsilon_{\text {radar }} / \varepsilon_{\text {sonic }}$ ) as a function of $\varepsilon_{\text {sonic }}$ (a) and as a function of $\varepsilon_{\text {radar }}(\mathbf{b})$, during April 2015 from the $449 \mathrm{MHz}$ (blue) and $915 \mathrm{MHz}$ (red) WPRs before applying the bias corrections (thin lines) and after, using the sonicdependent constant correction found from the month of March (thick lines).
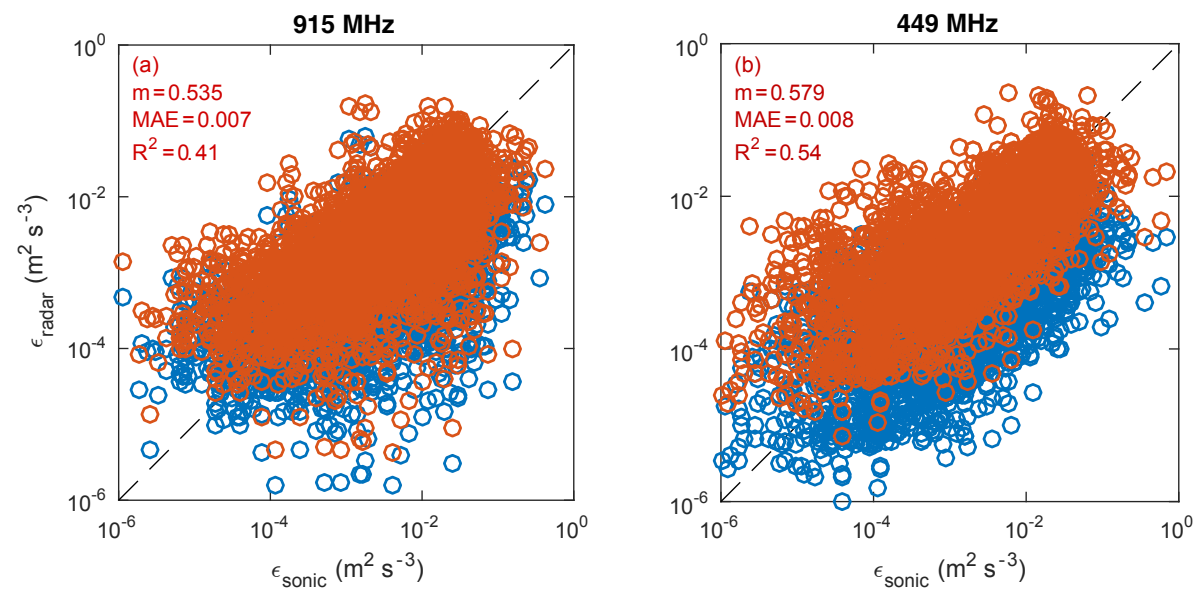

Figure 13. Observed dissipation rates, $\varepsilon$, from March and April from the $915 \mathrm{MHz}$ (a) and $449 \mathrm{MHz}$ (b) WPRs vs. the sonic anemometers at all overlapping heights, before (blue) and after (red) bias corrections using the sonic-dependent constant corrections. The black dashed lines are the one-to-one lines. Slope, $m$, mean absolute error, MAE, and coefficient of determination, $R^{2}$, of the bias-corrected (red) values are also shown.

Though the two corrections, based on each instrument, are not mathematically identical, their constant corrections are nearly the reciprocal of one another. This creates bias corrections that are nearly equal, but since the corrections are defined by an average, and applied to individual points before further averaging in Fig. 10, the results are not identical. The fact that they are so close, however, indicates that either correction method can be used.

Applying the same correction methods to the $449 \mathrm{MHz}$ WPR results in similarly improved dissipation rates, using $c_{1}=0.144$ and $c_{2}=5.114$, as seen in the fractional biases in Fig. 11. The bias correction makes larger improvements as a function of $\varepsilon_{\text {sonic }}$ (Fig. 11a), with more accurate dissipation rates measured down to $\varepsilon_{\text {sonic }}=10^{-4} \mathrm{~m}^{2} \mathrm{~s}^{-3}$. As a function of the WPR-estimated dissipation rate, the improvement is significant, removing all biases below $\varepsilon_{\text {radar }}=10^{-2} \mathrm{~m}^{2} \mathrm{~s}^{-3}$. There is no increase in the bias at the lowest dissipation rates, as was seen in the $915 \mathrm{MHz}$ WPR (Fig. 10b), showing that the $449 \mathrm{MHz}$ system is more accurate at these levels. 

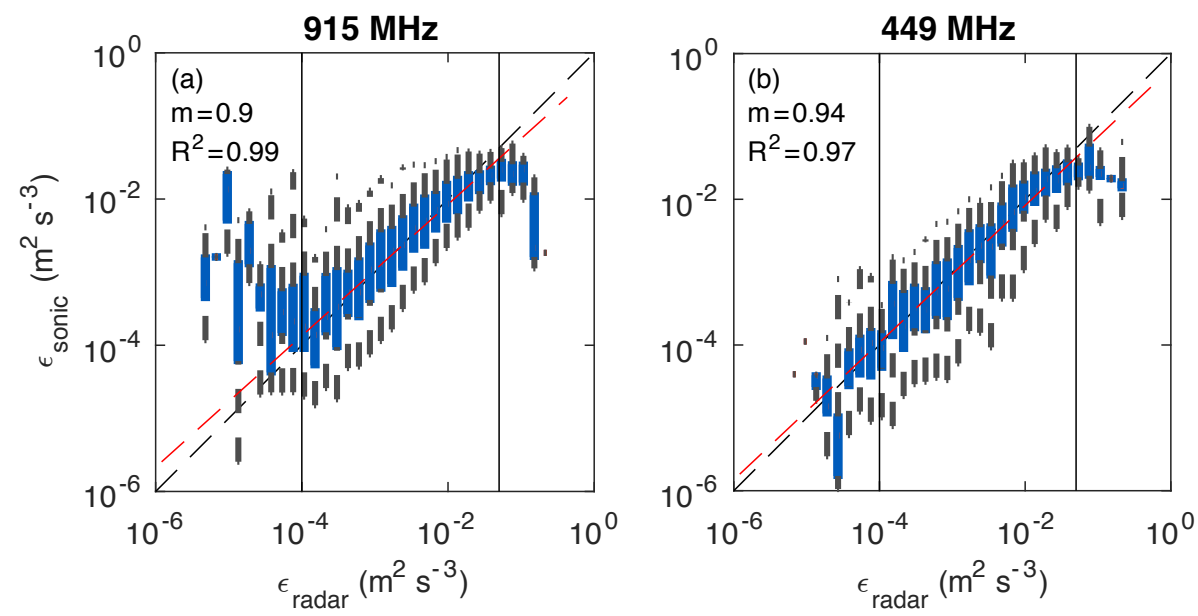

Figure 14. Box-and-whisker plots of observed dissipation rates, $\varepsilon$, of the sonic anemometers at all heights binned by the $915 \mathrm{MHz}$ (a) and $449 \mathrm{MHz}$ (b) WPR dissipation after using the sonic-dependent constant bias correction. Blue bars extend over the 25 th to 75 th percentiles, with dashed lines extending to the 90th percentiles. Red dashed lines are fit to the medians of each bin between the vertical lines at $10^{-4}$ and $5 \times 10^{-2} \mathrm{~m}^{2} \mathrm{~s}^{-3}$, with slope, $m$, labeled, as well as the $R^{2}$ values of the medians and bin centers in that range. The black dashed lines are the one-to-one lines.

These bias corrections were created based on 1 month of data (March) and show large improvements. However, the applicability of the corrections can be seen by applying the respective bias corrections for each radar to the observations taken during April. Figure 12 shows the remaining biases in the dissipation rates from the April datasets, using the sonic-dependent corrections found for the month of March. Both datasets show large improvements, particularly above $\varepsilon_{\text {sonic }}=3 \times 10^{-4} \mathrm{~m}^{2} \mathrm{~s}^{-3}$. When binned by $\varepsilon_{\text {radar }}$, the correction almost completely removes all biases. In Fig. 13, the sonic-dependent correction constants were used on each WPR, respectively, to adjust the entire 2 months of the original datasets (blue) to obtain the corrected data (red). The bias has been removed in the intermediate-to-large dissipation rates, and the scatter now falls on the one-to-one line, with MAE $=0.008 \mathrm{~m}^{2} \mathrm{~s}^{-3}$ for the $915 \mathrm{MHz}$ WPR and $\mathrm{MAE}=0.007 \mathrm{~m}^{2} \mathrm{~s}^{-3}$ for the $449 \mathrm{MHz}$ WPR.

\section{Uncertainty quantification}

Two different methods were used to quantify the uncertainty in the dissipation rates from the sonic anemometers and the WPRs. For the sonic anemometers, the error analysis method of Piper (2001) was applied, utilizing the standard deviation of the power spectrum in the inertial range over which the dissipation rates are estimated. The error in dissipation rate is given by

$\sigma_{\varepsilon}=\frac{3}{2} \varepsilon \frac{\sigma_{\mathrm{S}}}{S}$,

where $S$ is the mean and $\sigma_{\mathrm{S}}$ is the standard deviation of $f^{5 / 3} \phi_{\text {new }}(f)$ over the inertial range.
When investigating the accuracy of the bias-corrected WPR measurements, box-and-whisker plots were made from dissipation rates at all heights (Fig. 14), binned by $\varepsilon_{\text {radar }}$ (rather than $\varepsilon_{\text {sonic }}$ as in Figs. 6 and 8), to see the distribution of true dissipation rates (from sonic anemometers) for each WPR observation. This can serve as an error analysis for the dissipation rates estimated from the WPR spectral width. In each bin, the median and standard deviation were calculated, and the standard deviation will be used as the error for each radar measurement that falls in that bin. With the bias correction applied, the median dissipation rates of the sonic anemometers accurately match the radar value, with $R^{2}=0.99$ for the $915 \mathrm{MHz}$ and 0.97 for the $449 \mathrm{MHz}$ in the range of $\epsilon_{\text {radar }}=10^{-4}$ to $5 \times 10^{-2} \mathrm{~m}^{2} \mathrm{~s}^{-3}$, which contains the majority of the observed dissipation rates from the sonics. A fit to the medians in this range produces a line (red dashes) with slope, $m=0.9$ and 0.94 for the 915 and $449 \mathrm{MHz}$ WPRs, respectively. For the more accurate $449 \mathrm{MHz}$ WPR, only the highest measured $\varepsilon_{\text {radar }}$ values do not fall on the oneto-one line, but, as noted previously, these are infrequent observations. The smallest dissipations rates measured by the $915 \mathrm{MHz}$ WPR show more uncertainty, with broader ranges and medians that do not fall on the one-to-one line, showing that a measured value in this range is most likely to be underestimating the actual dissipation rate. It should also be noted that the constant bias correction factors for both WPRs are larger than 1 standard deviation from the corrected values $84 \%$ of the time for the $449 \mathrm{MHz}$ and $80 \%$ for the $915 \mathrm{MHz}$ WPR (for all heights), therefore being meaningful.

We also note that the lower $R^{2}$ values for the individual $30 \min \varepsilon$ values shown in Fig. 13 compared to the $R^{2}$ values of the binned medians in Fig. 14 are in part due to the $600 \mathrm{~m}$ separation between the BAO tower and the radars. If 


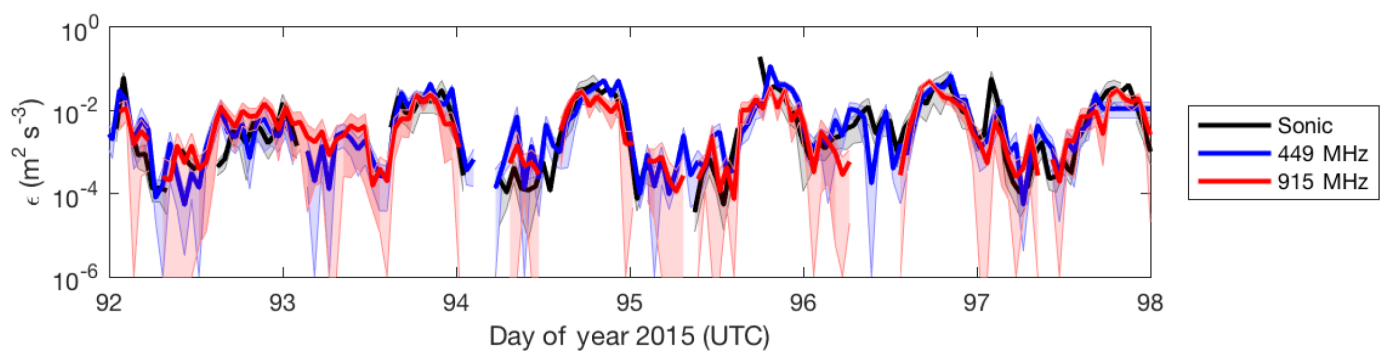

Figure 15. Dissipation rate, $\varepsilon$, from 2 to 8 April 2015 (hours in UTC), from the sonic anemometer (black) at $200 \mathrm{~m}$ and the $206 \mathrm{~m}$ range gate of the $449 \mathrm{MHz}$ WPR (blue) and the $201 \mathrm{~m}$ range gate of the $915 \mathrm{MHz}$ WPR (red) after bias corrections have been applied. Shaded regions signify 1 standard deviation from the mean, matching colors to the instruments.

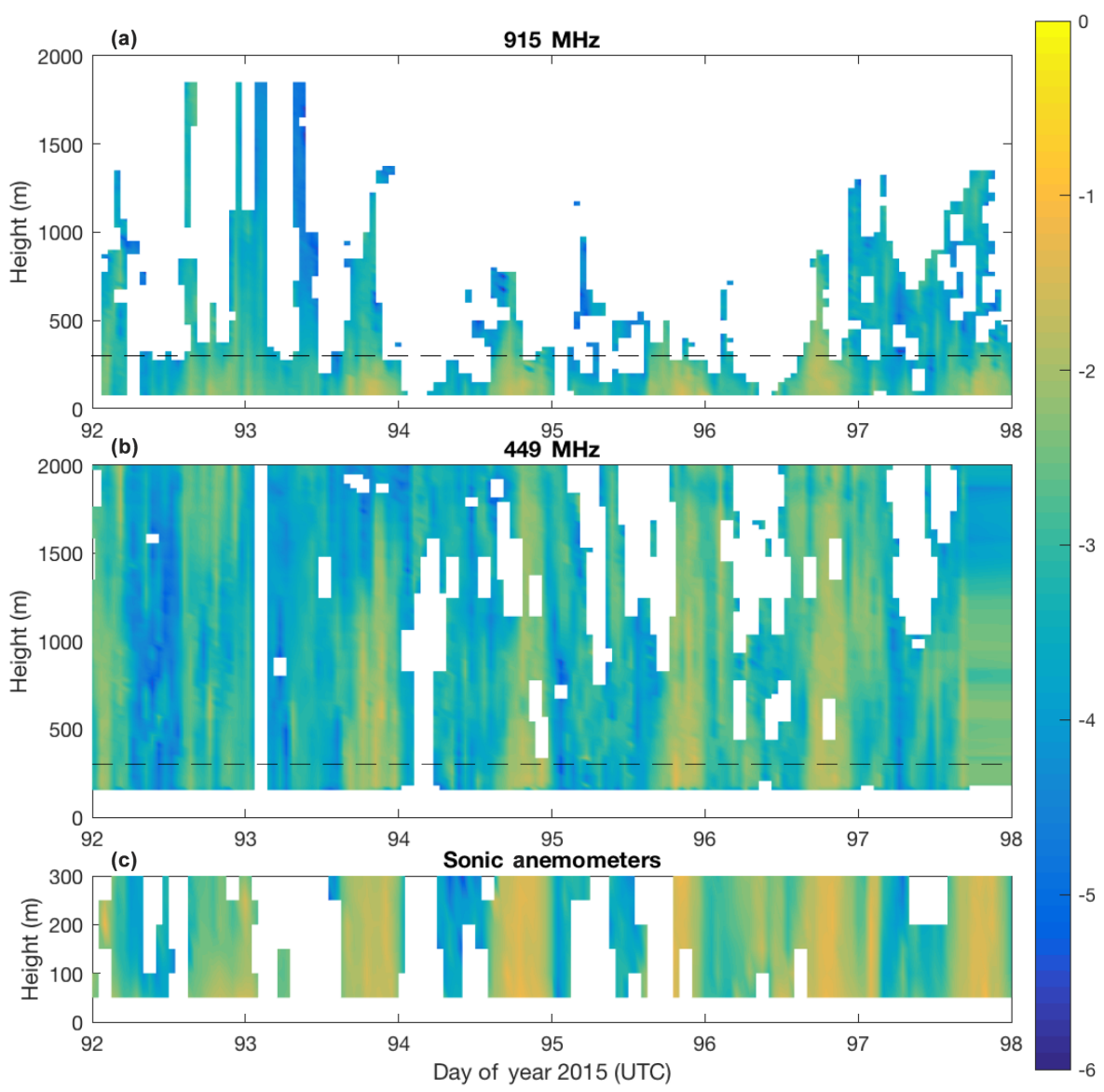

Figure 16. Dissipation rate, $\varepsilon$, from 2 to 8 April 2015 (hours in UTC), from the $915 \mathrm{MHz}$ (a) and $449 \mathrm{MHz}$ WPRs (b) and sonic anemometers on the $300 \mathrm{~m}$ tower (c). Height is in $\mathrm{m}$ a.g.l., and the dashed lines mark the top of the sonic anemometer measurements.

the WPRs had been located adjacent to the tower, the scatter in Fig. 13 would likely have been smaller.

An example time series of bias-corrected dissipation rates near $200 \mathrm{~m}$ from both WPRs and sonic anemometers is shown in Fig. 15, with a range of 1 standard deviation shown for each instrument. The diurnal cycle is evident, and is consistently captured by both WPR systems, within the error bounds of both instruments. Several instances of sharp increases in dissipation rate (such as at the beginning of day
97) are matched well by the WPRs and sonics, highlighting the ability of the WPR to capture turbulent events in the PBL. Full profiles of the dissipation rates are presented in Fig. 16. The growth of the PBL is visible in both datasets, with lower values during nighttimes and increasing dissipation rates in the morning hours of each day. The $449 \mathrm{MHz}$ WPR, with its higher SNR, is able to observe more consistent profiles up to $2 \mathrm{~km}$, while the $915 \mathrm{MHz}$ WPR is limited by its lower SNR, and only when the spectral widths (and subsequent dis- 
sipation rates) are large enough are the observations possible. This occurs most consistently in the bottom $1 \mathrm{~km}$ of the PBL but occasionally up to $1500 \mathrm{~m}$.

\section{Conclusions}

Using an optimized setup of two WPRs during the XPIA field campaign in March and April 2015, turbulence dissipation rates were calculated and compared to in situ observations from sonic anemometers on the $300 \mathrm{~m}$ tower at the BAO. Using only the vertically pointing beam and a large number of FFT points to obtain the Doppler spectra with high spectral resolution, post-processing methods were compared to determined the optimal method of estimating dissipation rates from the WPRs. The MPP method of calculating spectral moments showed inaccurate results, often measuring spectral widths that were far too small, most likely due to MPP only selecting part of the total atmospheric peak at high spectral resolution. Using the maximum or mean noise level with the SPP method showed small differences, but ultimately the mean noise level was chosen since it produced lower biases in dissipation rates than the maximum noise level. Analysis of the dwell time, dependent on the number of spectral averages, showed that, for both the 915 and $449 \mathrm{MHz}$ WPRs, dwell times of approximately 2 min $(\mathrm{NSPEC}=8)$ produced the most accurate dissipation rates. Dwell times of the unaveraged spectra $($ NSPEC $=1)$ have insufficiently low SNRs, but averaging too long widens the Doppler spectral peak with nonstationary turbulence. This timescale supports the similar results of McCaffrey et al. (2017a) based on vertical velocity variance from spectral widths.

A simple bias correction was applied to the WPR dissipation rates, based on the fractional bias between the radarestimated and sonic-estimated dissipation rates. A slightly smaller correction was needed for the $915 \mathrm{MHz}$ WPR, and the constant correction produced improved dissipation rates above values of $\varepsilon_{\text {sonic }}=6 \times 10^{-4} \mathrm{~m}^{2} \mathrm{~s}^{-3}$, reducing the MAE from $9 \times 10^{-3}$ to $8 \times 10^{-3} \mathrm{~m}^{2} \mathrm{~s}^{-3}$. For the $449 \mathrm{MHz}$ WPR, the full range of values of dissipations rates was improved through a similar constant bias correction, reducing the MAE from $9 \times 10^{-3}$ to $8 \times 10^{-3} \mathrm{~m}^{2} \mathrm{~s}^{-3}$. With the bias corrections applied, time series of the dissipation rates from the two WPRs compared well (especially the $449 \mathrm{MHz}$ WPR) with sonic anemometers, with the entire range of dissipation rates captured throughout the diurnal cycle. The resulting coefficients of determination between the logarithms of dissipation rates are $R^{2}=0.41$ for the $915 \mathrm{MHz}$ WPR and 0.54 for the $449 \mathrm{MHz}$ WPR. The bias corrections were determined for each radar based on 1 month of data, with a second month used to test the applicability of the corrections. Other datasets could provide additional validation of usefulness of the correction for other radar systems or different times of the year, but the results herein are encouraging. The $R^{2}$ values were adversely impacted by the $600 \mathrm{~m}$ separation between the WPRs and the tower. $R^{2}$ values for bin-averaged data that removes the spatial separation effect are as high as 0.99 for the $915 \mathrm{MHz}$ WPR and 0.97 for the $449 \mathrm{MHz}$ WPR.

High-vertical-resolution profiles of dissipation rates up to $2 \mathrm{~km}$ are obtainable from the $449 \mathrm{MHz}$ WPR and often up to $1 \mathrm{~km}$ from the $915 \mathrm{MHz}$ WPR. These observations will be very useful for the validation of boundary layer parameterizations in numerical weather prediction models and reduce the uncertainty due to these parameters as seen in Yang et al. (2016). While profiling lidars and sodars can provide turbulence measurements in the lower levels of the boundary layer (Ottersten et al., 1973; Smalikho and Banakh, 2013), WPRs can supply these measurements to higher levels, above the PBL. Future studies could more closely collocate a WPR with sonic anemometers to analyze the shorter timescales that could possibly be captured by the spectral widths of the WPR measurement volume. Those more detailed observations would allow for analysis of the development and degradation of the convection boundary layer throughout day.

Data availability. Data are available at the A2E Data Archive and Portal at http://a2e.energy.gov/data\#xpia.

Author contributions. KM completed the primary analysis with the aid of LB and JW. KM prepared the manuscript with contributions from all co-authors.

Special issue statement. This article is part of the special issue "Pushing the limits: The eXperimental Planetary boundary layer Instrumentation Assessment (XPIA) campaign". It is not associated with a conference.

Acknowledgements. Thanks are due to Timothy Coleman, Daniel Gottas, Paul Johnston, and Dave Carter for their role in data acquisition and post-processing. Katherine McCaffrey was funded by the NRC Postdoctoral Fellowship. The XPIA field program was funded under the US Department of Energy's Atmospheres to Electrons (A2e) program and by NOAA/ESRL. We would like to acknowledge operational, technical, and scientific support for the sonic anemometry provided by NCAR's Earth Observing Laboratory's CABL project, sponsored by the National Science Foundation.

Edited by: Julie Lundquist

Reviewed by: two anonymous referees 


\section{References}

Angevine, W. M., Doviak, R. J., and Sorbjan, Z.: Remote sensing of vertical velocity variance and surface heat flux in a convective boundary layer, J. Appl. Meteorol., 33, 977-983, 1994.

Champagne, F. H.: The fine-scale structure of the turbulent velocity field, J. Fluid Mech., 86, 67-108, 1978.

Cohn, S. A.: Radar measurements of turbulent eddy dissipation rate in the troposphere: A comparison of techniques, J. Atmos. Ocean. Tech., 12, 85-95, 1995.

Dehghan, A., Hocking, W. K., and Srinivasan, R.: Comparisons between multiple in-situ aircraft turbulence measurements and radar in the troposphere, J. Atmos. Sol.-Terr. Phy., 118, 64-77, 2014

Frehlich, R., Meillier, Y., Jensen, M. L., and Balsley, B.: Turbulence measurements with the CIRES tethered lifting system during CASES-99: Calibration and spectral analysis of temperature and velocity, J. Atmos. Sci., 60, 2487-2495, 2003.

Frisch, A. and Clifford, S.: A study of convection capped by a stable layer using Doppler radar and acoustic echo sounders, J. Atmos. Sci., 31, 1622-1628, 1974.

Gaffard, C., Bianco, L., Klaus, V., and Matabuena, M.: Evaluation of moments calculated from wind profiler spectra: A comparison between five different processing techniques, Meteorol. Z., 15, 73-85, 2006.

Gossard, E.: Radar research on the atmospheric boundary layer, Radar in Meteorology, 477-527, 1990.

Gossard, E., Wolfe, D., Moran, K., Paulus, R., Anderson, K., and Rogers, L.: Measurement of clear-air gradients and turbulence properties with radar wind profilers, J. Atmos. Ocean. Tech., 15, 321-342, 1998.

Hocking, W. K.: Measurement of turbulent energy dissipation rates in the middle atmosphere by radar techniques: A review, Radio Sci., 20, 1403-1422, 1985.

Jacoby-Koaly, S., Campistron, B., Bernard, S., Bénech, B., Ardhuin-Girard, F., Dessens, J., Dupont, E., and Carissimo, B.: Turbulent dissipation rate in the boundary layer via UHF wind profiler Doppler spectral width measurements, Bound.-Lay. Meteorol., 103, 361-389, 2002.

Jordan, J. R., Lataitis, R. J., and Carter, D. A.: Removing ground and intermittent clutter contamination from wind profiler signals using wavelet transforms, J. Atmos. Ocean. Tech., 14, 1280-1297, 1997.

Kaimal, J.: Sonic anemometer measurement of atmospheric turbulence, in: Proceedings of the Dynamic Flow Conference 1978 on Dynamic Measurements in Unsteady Flows, 551-565, Springer, 1978.

Kaimal, J., Wyngaard, J., and Haugen, D.: Deriving power spectra from a three-component sonic anemometer, J. Appl. Meteorol., 7, 827-837, 1968.

Kolmogorov, A. N.: The local structure of turbulence in incompressible viscous fluid for very large Reynolds numbers, in: Dokl. Akad. Nauk SSSR, 30, 299-303, available at: http://www. jstor.org/stable/pdf/51980.pdf, 1941.

Labbitt, M.: Coordinated radar and aircraft observations of turbulence Rep, ATC-108, 40 Lincoln Lab., Mass. Inst. of Technol., Cambridge, 1981.

Lehmann, V.: Optimal Gabor-frame-expansion-based intermittentclutter-filtering method for radar wind profiler, J. Atmos. Ocean. Tech., 29, 141-158, 2012.
Lemone, M. A. and Pennell, W. T.: A comparison of turbulence measurements from aircraft, J. Appl. Meteorol., 19, 1420-1437, 1980.

Lundquist, J. K., Wliczak, J. M., Ashton, R., Bianco, L., Brewer, W. A., Choukulkar, A., Clifton, A. J., Debnath, M., Delgado, R., Friedrich, K., Gunter, S., Hamidi, A., Iungo, G. V., Kaushik, A., Kosovik, B., Langan, P., Lass, A., Lavin, E., Lee, J. C.-Y., Newsom, R. K., Noone, D. C., Oncley, S. P., Quelet, P. T., Sandberg, S. P., Schroeder, J. L., Shaw, W. J., Sparling, L., St. Martin, C., St. Pe, A., Strobach, E., Tay, K., Vanderwende, B. J., Weickmann, A., Wolfe, D., and Worsnop, R.: Assessing state-ofthe-art capabilities for probing the atmospheric boundary layer: the XPIA field campaign, B. Am. Meteorol. Soc., 98, 289-314, https://doi.org/10.1175/BAMS-D-15-00151.1, 2016.

McCaffrey, K., Bianco, L., Johnston, P., and Wilczak, J. M.: A comparison of vertical velocity variance measurements from wind profiling radars and sonic anemometers, Atmos. Meas. Tech., 10, 999-1015, https://doi.org/10.5194/amt-10-999-2017, 2017.

McCaffrey, K., Quelet, P. T., Choukulkar, A., Wilczak, J. M., Wolfe, D. E., Oncley, S. P., Brewer, W. A., Debnath, M., Ashton, R., Iungo, G. V., and Lundquist, J. K.: Identification of tower-wake distortions using sonic anemometer and lidar measurements, Atmos. Meas. Tech., 10, 393-407, https://doi.org/10.5194/amt-10393-2017, 2017.

Nastrom, G.: Doppler radar spectral width broadening due to beamwidth and wind shear, Annales Geophysicae, 15, 786-796, Springer, 1997.

Nastrom, G. and Eaton, F.: Turbulence eddy dissipation rates from radar observations at $5-20 \mathrm{~km}$ at White Sands Missile Range, New Mexico, J. Geophys. Res.-Atmos., 102, 19495-19505, 1997.

Nicholls, S.: Measurements of turbulence by an instrumented aircraft in a convective atmospheric boundary layer over the sea, Q. J. Roy. Meteor. Soc., 104, 653-676, 1978.

Oncley, S. P., Friehe, C. A., Larue, J. C., Businger, J. A., Itsweire, E. C., and Chang, S. S.: Surface-layer fluxes, profiles, and turbulence measurements over uniform terrain under near-neutral conditions, J. Atmos. Sci., 53, 1029-1044, 1996.

Ottersten, H., Hardy, K. R., and Little, C. G.: Radar and sodar probing of waves and turbulence in statically stable clear-air layers, Bound.-Lay. Meteorol., 4, 47-89, 1973.

Piper, M.: The effects of a frontal passage on fine-scale nocturnal boundary layer turbulence, $\mathrm{PhD}$ thesis, University of Colorado, 2001.

Riddle, A. and Angevine, W.: Ground clutter removal from profiler spectra, in: Solar-Terrestrial Energy Program, 1, p. 418, 1991.

Riddle, A. C., Hartten, L. M., Carter, D. A., Johnston, P. E., and Williams, C. R.: A minimum threshold for wind profiler signalto-noise ratios, J. Atmos. Ocean. Tech., 29, 889-895, 2012.

Sathe, A. and Mann, J.: A review of turbulence measurements using ground-based wind lidars, Atmos. Meas. Tech., 6, 3147-3167, https://doi.org/10.5194/amt-6-3147-2013, 2013.

Shaw, W. J. and LeMone, M. A.: Turbulence dissipation rate measured by $915 \mathrm{MHz}$ wind profiling radars compared with in-situ tower and aircraft data, in: 12th Symposium on Meteorological Observations and Instrumentation, American Meteorological Society, California, available at: https://ams.confex.com/ams/ pdfpapers/58647.pdf, 2003. 
Smalikho, I. and Banakh, V.: Accuracy of estimation of the turbulent energy dissipation rate from wind measurements with a conically scanning pulsed coherent Doppler lidar - Part I: Algorithm of data processing, Atmos. Ocean. Opt., 26, 404-410, 2013.

Tennekes, H. and Lumley, J. L.: A first course in turbulence, MIT press, 1972.

White, A., Lataitis, R., and Lawrence, R.: Space and time filtering of remotely sensed velocity turbulence, J. Atmos. Ocean. Tech., 16, 1967-1972, 1999.

Wilczak, J. M., Oncley, S. P., and Stage, S. A.: Sonic anemometer tilt correction algorithms, Bound.-Lay. Meteorol., 99, 127-150, 2001.
Wyngaard, J. and Clifford, S.: Taylor's hypothesis and highfrequency turbulence spectra, J. Atmos. Sci., 34, 922-929, 1977.

Yang, B., Qian, Y., Berg, L. K., Ma, P.-L., Wharton, S., Bulaevskaya, V., Yan, H., Hou, Z., and Shaw, W. J.: Sensitivity of Turbine-Height Wind Speeds to Parameters in Planetary Boundary-Layer and Surface-Layer Schemes in the Weather Research and Forecasting Model, Bound.-Lay. Meteorol., 162, 117142, 10.1007/s10546-016-0185-2, 2016. 